\title{
Macrophage AXL receptor tyrosine kinase inflames the heart after reperfused myocardial infarction
}

\author{
Matthew DeBerge, ${ }^{1,2}$ Kristofor Glinton, ${ }^{1,2}$ Manikandan Subramanian, ${ }^{3}$ Lisa D. Wilsbacher, ${ }^{2,4}$ Carla V. Rothlin, ${ }^{5}$ \\ Ira Tabas, ${ }^{6}$ and Edward B. Thorp ${ }^{1,2}$ \\ 'Department of Pathology and ${ }^{2}$ Feinberg Cardiovascular and Renal Research Institute, Feinberg School of Medicine, Northwestern University, Chicago, Illinois, USA. ${ }^{3}$ William Harvey Research Institute, Barts \\ and The London School of Medicine and Dentistry, Queen Mary University of London, London, United Kingdom. ${ }^{4}$ Department of Medicine, Feinberg School of Medicine, Northwestern University, Chicago, \\ Illinois, USA. 5Department of Immunobiology, Yale School of Medicine, New Haven, Connecticut, USA. ${ }^{5}$ Departments of Medicine, Pathology and Cell Biology, and Physiology, Columbia University Irving \\ Medical Center, New York City, New York, USA
}

\begin{abstract}
Tyro3, AXL, and MerTK (TAM) receptors are activated in macrophages in response to tissue injury and as such have been proposed as therapeutic targets to promote inflammation resolution during sterile wound healing, including myocardial infarction. Although the role of MerTK in cardioprotection is well characterized, the unique role of the other structurally similar TAMs, and particularly AXL, in clinically relevant models of myocardial ischemia/reperfusion infarction (IRI) is comparatively unknown. Utilizing complementary approaches, validated by flow cytometric analysis of human and murine macrophage subsets and conditional genetic loss and gain of function, we uncover a maladaptive role for myeloid AXL during IRI in the heart. Cross signaling between AXL and TLR4 in cardiac macrophages directed a switch to glycolytic metabolism and secretion of proinflammatory IL-1ß, leading to increased intramyocardial inflammation, adverse ventricular remodeling, and impaired contractile function. AXL functioned independently of cardioprotective MerTK to reduce the efficacy of cardiac repair, but like MerTK, was proteolytically cleaved. Administration of a selective small molecule AXL inhibitor alone improved cardiac healing, which was further enhanced in combination with blockade of MerTK cleavage. These data support further exploration of macrophage TAM receptors as therapeutic targets for myocardial infarction.
\end{abstract}

\section{Introduction}

Myocardial infarction (MI) is a leading cause of heart failure and is associated with an increased risk of death. Intramyocardial inflammation arising after cardiomyocyte cell death from reperfused infarction contributes to pathological cardiac remodeling and progression to heart failure $(1,2)$. Phagocytes within the heart, particularly macrophages, recognize damage-associated molecular patterns (DAMPs) released by dying cardiomyocytes, which triggers pathological inflammatory responses (3). Although results from clinical trials with broad immunosuppression have failed to show any effect (4), specific targeting of proinflammatory cytokines has conferred clinical benefits (2). Recent results from the Canakinumab Anti-Inflammatory Thrombosis Outcome Study (CANTOS, ref. 5), which targeted the inflammatory cytokine interleukin $1 \beta$ (IL-1 $\beta$ ), demonstrated the merits of a targeted approach, as opposed to generalized immunosuppression, in reducing recurrent cardiovascular events in patients with ischemic cardiomyopathy, albeit with a compromise in host defense.

Macrophages express a family of receptor tyrosine kinases, Tyro3, AXL, and MerTK (TAM), that mediate the clearance of apoptotic cells (efferocytosis), regulate inflammatory cyto-

Conflict of interest: The authors have declared that no conflict of interest exists. Copyright: () 2021, American Society for Clinical Investigation.

Submitted: April 29, 2020; Accepted: January 27, 2021; Published: March 15, 2021

Reference information: J Clin Invest. 2021;131(6):e139576.

https://doi.org/10.1172/JCl139576. kine production, and in the case of MerTK, mediate resolution of inflammation (6-9). Although the role of Mertk- and phagocytosis-dependent cardiac repair after MI is well characterized (10-12), less is known about the role of other TAM receptors in tissue repair after myocardial injury. AXL protein expression is increased in myocardial biopsies of patients with end-stage heart failure (13). Moreover, the extracellular domain of AXL is proteolytically cleaved, which releases a stable product called soluble AXL (solAXL), and there is a correlation among elevated solAXL, heightened markers of inflammation, and poor prognosis during heart failure $(13,14)$. However, whether AXL or its cleavage regulates the initial inflammatory and reparative macrophage responses in a manner that affects disease progression, either positively or negatively, requires investigation.

To ascertain the role of macrophage AXL and AXL cleavage during ischemic tissue injury, we examined cardiac repair in mice genetically engineered to lack AXL or to be resistant to AXL cleavage. Our findings reveal that AXL, in contrast to MerTK, antagonizes optimal cardiac repair by promoting a switch to glycolytic metabolism in macrophages to fuel proinflammatory responses and that AXL cleavage is a mitigating factor in this process.

\section{Results}

We first evaluated AXL expression on myeloid cells in mice subjected to a clinically relevant model of myocardial ischemia/reperfusion infarction (IRI). Both gene and protein expression of AXL was detected in a subset of cardiac macrophages expressing high levels of major 
A

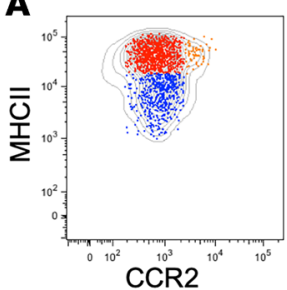

C

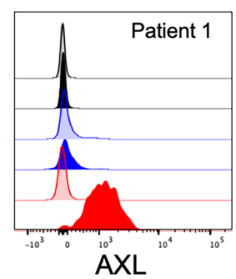

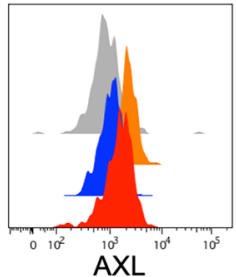

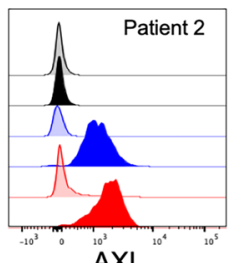

AXL
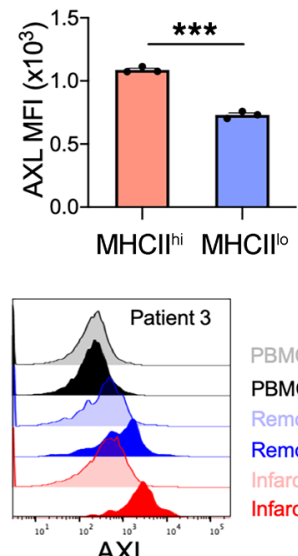

AXL
B
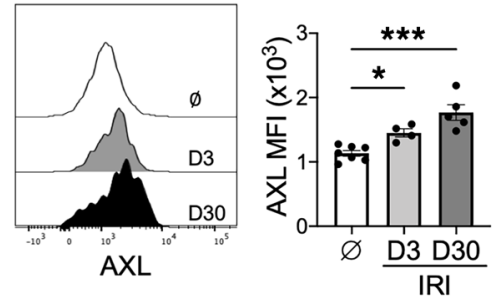

Figure 1. AXL expression is increased on human and murine macrophages after myocardial ischemia/reperfusion infarction (IRI). (A) Cell-surface protein

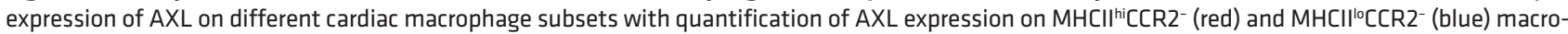
phages. The gray histogram represents $A x^{l_{-1}}$ cardiac macrophage staining control. Data are representative of 3 independent experiments. $n=3$ mice/group. ${ }^{* * *} P<0.001$ by 2-tailed, unpaired $t$ test. (B) AXL expression on murine cardiac macrophages before (Ø) and 3 or 30 days after myocardial IRI. $n=4-7$ mice / group pooled from 2 independent experiments. ${ }^{*} P<0.05$, ${ }^{* *} P<0.001$ by 1-way ANOVA followed by Tukey's test. (C) Expression of AXL on peripheral blood mononuclear cells (PBMCs) or human cardiac macrophages isolated from peri-infarct or remote tissue from the explanted hearts of patients with ischemic cardiomyopathy at the time of heart transplantation. Fluorescence minus one (FMO) was used as a staining control. All data presented as mean \pm SEM.

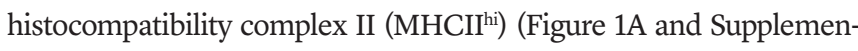
tal Figure 1, A and B; supplemental material available online with this article; https://doi.org/10.1172/JCI139576DS1). Survey of tissue-resident macrophages across various tissues demonstrated widespread expression of AXL on macrophages, with preferential AXL expression on $\mathrm{MHCII}^{\mathrm{hi}}$ subsets in a variety of tissues, including the peritoneum and liver (Supplemental Figure 2), consistent with data assembled by the ImmGen consortium (15) and previous reports $(16,17)$. In contrast, AXL protein expression was not detectable on either neutrophils or Ly6 $\mathrm{C}^{\text {hi }}$ monocytes (Supplemental Figure 1, C and D), indicating that $\mathrm{MHCII}^{\mathrm{hi}}$ cardiac macrophages were a major source for myeloid cell AXL in the heart. Following IRI, AXL expression was increased on infarct-associated $\mathrm{MHCII}^{\mathrm{hi}}$ cardiac macrophages and remained significantly increased through the progression to heart failure 30 days after IRI (Figure 1B). Thus, macrophage AXL expression persists after IRI through the progression to heart failure. Moreover, a relatively high level of AXL expression was detected in macrophages obtained from peri-infarct tissue of explanted hearts in patients with ischemic cardiomyopathy (Figure 1C and Supplemental Figure 3).

To determine the causal functional role of AXL expression in cardiac macrophages in the acute phase after IRI, we compared left ventricular (LV) remodeling and contractile function between $A x l^{+/+}$and $A x l^{--}$mice after IRI. Pathological measurements of infarct size 7 days after IRI revealed smaller-sized infarcts in $A x^{-/}$mice compared with $A x^{+++}$mice (Figure 2A). Despite similar baseline echocardiographic parameters, $A x l$ deficiency resulted in greater preservation of LV systolic function 21 days after IRI compared with $\mathrm{Axl}^{+++}$mice (Figure 2B). Given that AXL is also expressed on nonmyeloid cells, including endothelial cells and dendritic cells $(18,19)$, we performed IRIs on lethally irradiated WT recipients of $A x l$-deficient bone marrow or in mice with selective $A x l$ deletion in myeloid cells $\left(L y s M-C r e^{+} A x l^{l / f l}\right.$ mice, Figure 2C). Similarly to whole-body knockouts, myeloid-specific deletion of Axl resulted in significantly smaller infarcts 7 days after IRI compared with controls (Figure 2D and Supplemental Figure 4A) and these results extended to both female mice and a small cohort of male mice (Supplemental Figure 4B). Because LysM-Cre-mediated recombination results in $A x l$ deletion predominantly among monocytes, neutrophils, and macrophages and AXL was not detected on monocytes and neutrophils, these results suggest that AXL acts in macrophages to exacerbate cardiac repair after IRI.

We next investigated whether $A x l$ deficiency impacted the extent and quality of immune cell infiltrate in the heart after IRI. Using flow cytometry, we found that $A x l$ deficiency did not affect initial neutrophil and $\mathrm{Ly} 6 \mathrm{C}^{\text {hi }}$ monocyte recruitment to the infarcted myocardium (Figure 3, A and B). However, by 3 days after IRI, there was a significant reduction in the total number of neutrophils and Ly6 $\mathrm{C}^{\text {hi }}$ monocytes in $A x t^{--}$mice compared with $A x^{l^{+/}}$mice (Figure 3 , A and B). Importantly, similar total number of neutrophils and Ly6 $\mathrm{C}^{\text {hi }}$ monocytes were observed in the periphery of $A \mathrm{xl}^{\mathrm{t}^{++}}$and $\mathrm{Ax \textrm {t } ^ { - / }}$ mice at both baseline and after IRI (Supplemental Figure 5), consistent with a role for AXL in amplifying local cardiac inflammation. Although no differences were observed in the total number of macrophages in the infarcted myocardium after IRI (Figure 3C), there was an increase in macrophage polarization toward a reparative phenotype in $\mathrm{Axl}^{-/}$mice compared with $A \mathrm{xl}^{+/+}$mice, as measured by an increase in the ratio of $\mathrm{MHCII}^{\mathrm{lo}}$ to $\mathrm{MHCII}^{\text {hi }}$ cardiac macrophages (Figure 3D). We and others have shown that $\mathrm{MHCII}^{\mathrm{lo}}$ cardiac macrophages secrete higher levels of IL-10 to restrain inflammation and fibrosis $(12,20)$, while $\mathrm{MHCII}^{\mathrm{hi}}$ cardiac macrophages are enriched in genes regulating the NLRP3 inflammasome (21), which regulates IL-1 $\beta$ secretion. Despite similar baseline levels, we observed increased expression of antiinflammatory genes, $I l 10$ and $T g f b$, and decreased proinflammatory genes, Illb, Il6, and Tnf, within cardiac extracts (Figure 3E), as well as decreased IL-1 $\beta$ and increased IL-10 levels in the serum of $A x^{-/}$mice compared with $A x^{l^{+/+}}$mice (Figure 
A

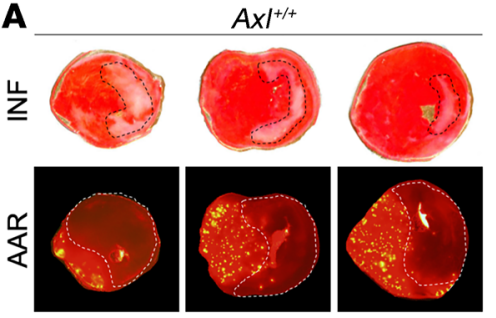

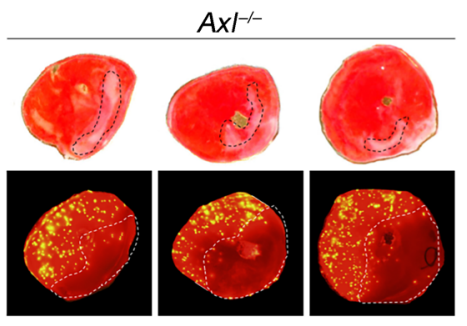
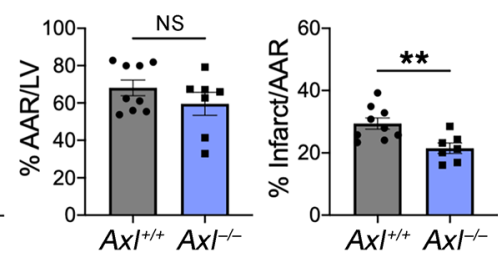

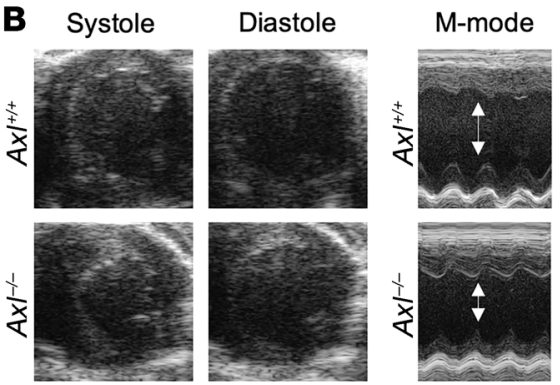

C
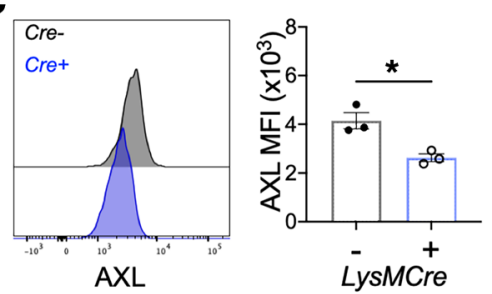

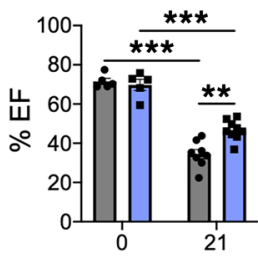

Days after IRI

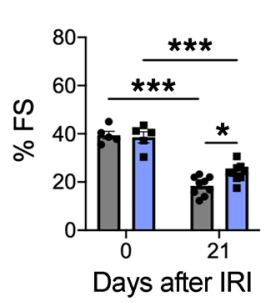

D

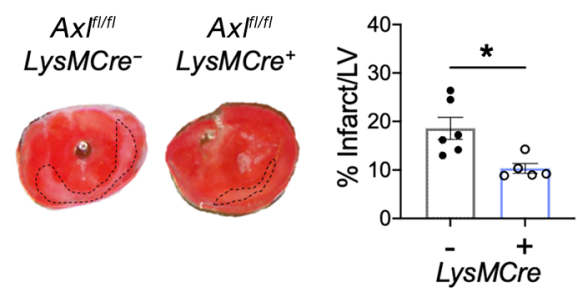

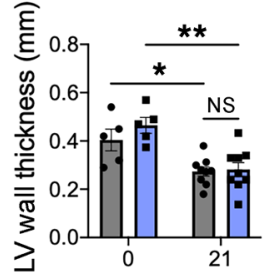

Days after IRI
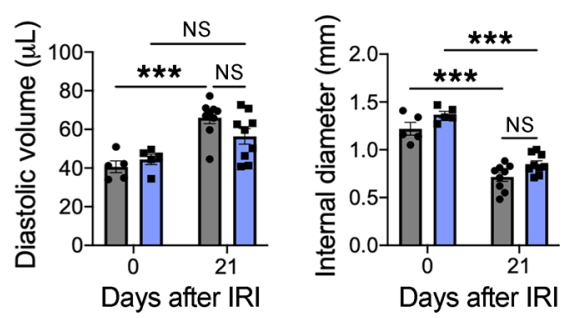

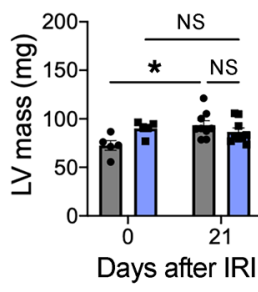

- $A x l^{+++}$

- $\left.A x\right|^{-1}$

Figure 2. Macrophage AXL worsens cardiac repair after myocardial ischemia/reperfusion infarction (IRI). (A) Representative 1-mm heart sections from an individual $\mathrm{Axl}^{+/+}$or $A \mathrm{xl}^{-/-}$mouse 7 days after IRI stained with triphenyltetrazolium chloride (TTC) for infarct measurements or injected with fluorescent microspheres to quantify the area at risk (AAR). From left to right, apex toward the ligation site. Percentage infarct/left ventricle (LV), percentage AAR/LV, and percentage infarct/AAR measured 7 days after IRI in mice with whole-body deletion of $A x I . n=7-9$ mice/group pooled from 3 independent experiments. ${ }^{*} P<0.05,{ }^{* *} P<0.01$ by 2 -tailed, unpaired $t$ test. (B) Representative B-mode and $\mathrm{M}$-mode echocardiography images of systole and diastole in hearts 21 days after IRI with quantification of percentage ejection fraction (\% EF), percentage fractional shortening (\% FS), systolic and diastolic volume, LV wall thickness, internal diameter, and LV mass 21 days after IRI. $n=5-9$ mice/group pooled from 2 independent experiments. ${ }^{*} P<0.05$, ${ }^{* *} P<0.01$, ${ }^{* *} P<0.001$ by 2-way ANOVA followed by Tukey's test. (C) AXL expression on cardiac macrophages in mice with myeloid-specific deletion of $A x I$ (LysM$\left.\mathrm{Cre}^{+} A \mathrm{x}^{|f / f|}\right)$ as measured by flow cytometry. Data are representative of 2 independent experiments. $n=3$ mice/group. ${ }^{*} P<0.05$ by 2 -tailed, unpaired $t$ test. (D) Infarct measurements 7 days after IRI in $L y s M-C r e^{+} A x^{f \mid l f f l}$ mice compared with LysM-Cre- $A x^{|f| f \mid}$ littermate controls. $n=5-6$ mice/group pooled from 2 independent experiments. ${ }^{*} P<0.05$ by 2 -tailed, unpaired $t$ test. All data presented as mean \pm SEM.

3F). Similarly to whole-body knockouts, myeloid-specific deletion of $\mathrm{Axl}$ using $\mathrm{LysM} \mathrm{M}-\mathrm{Cr} e^{+} \mathrm{Ax} \mathrm{l}^{\mathrm{l} / \mathrm{l} l}$ mice resulted in increased reparative MHCII ${ }^{\mathrm{lo}}$ macrophages, which was associated with a decrease in inflammatory neutrophils and monocytes within the infarct and reduced serum levels of IL-1 $\beta$ (Supplemental Figure 6). Axl deficiency also favored $\mathrm{MHCII}^{\mathrm{lo}}$ macrophage polarization in a model of peritonitis (Supplemental Figure 7), suggesting that AXL signaling promotes inflammatory reprogramming of macrophages in a variety of inflammatory settings.

Next, we explored how AXL signaling in macrophages promoted inflammatory reprogramming after IRI. To assess macrophage polarization, we examined proinflammatory gene expression in bone marrow-derived macrophages (BMDMs) from $\mathrm{Axl}^{+/+}$and
Axt $\mathrm{H}^{-\sim}$ mice after stimulation of Toll-like receptor 4 (TLR4), which recognizes DAMPs released by dying cardiomyocytes (22). Similarly to our findings in vivo, $A x l$ deficiency attenuated expression of proinflammatory genes, including Il1b and $T n f$, and increased antiinflammatory Il1O in BMDMs after TLR4 stimulation without affecting baseline gene levels (Figure 4A). Importantly, both $A x^{l^{++}}$and $A x^{l^{--}}$BMDMs expressed similar levels of TLR4 (Figure $4 \mathrm{~B})$. Given the recent success in targeting IL-1 $\beta$ to reduce recurrent MIs in ischemic cardiomyopathy patients (5), we performed additional experiments to investigate the relationship between AXL signaling and macrophage production of IL-1 $\beta$. In serumstarved BMDMs, addition of AXL-specific agonists, including either growth arrest-specific 6 (Gas6) or an agonizing antibody, 
A

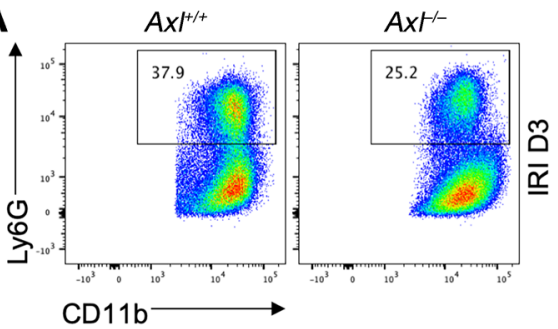

C

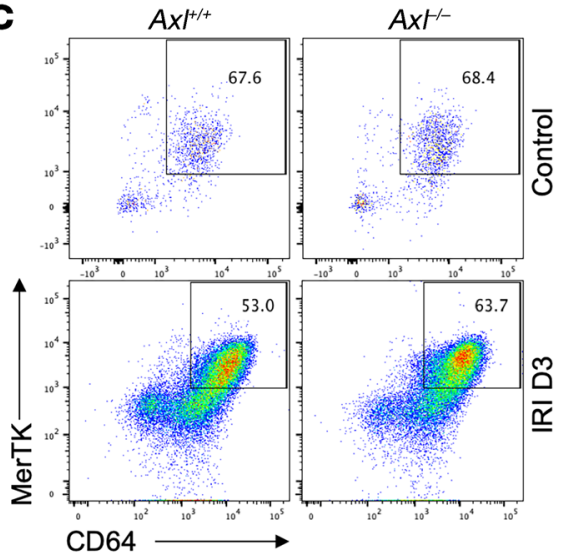

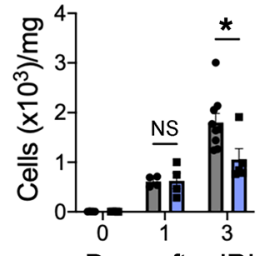

Days after IRI

B

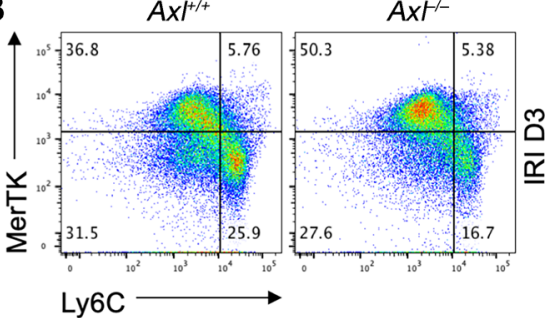

Ly6C

D

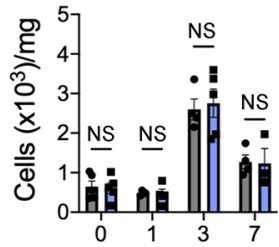

Days after IRI

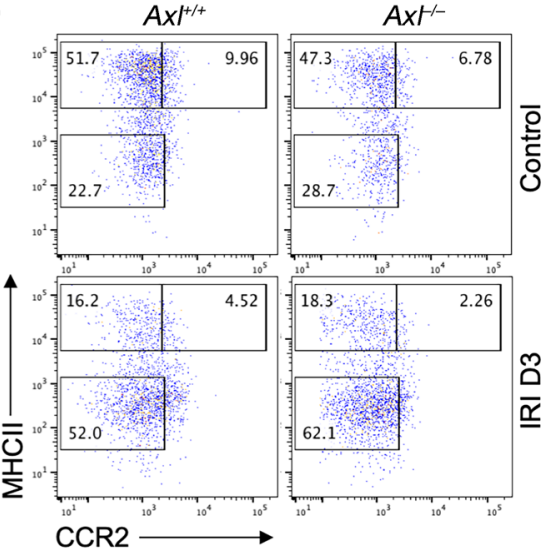

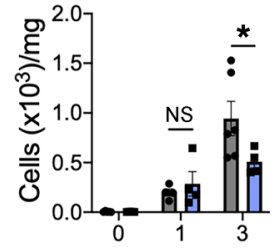

Days after IRI

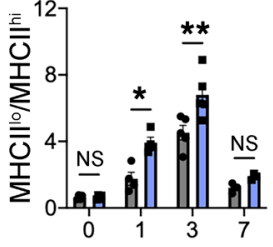

Days after IRI

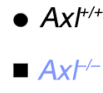

E

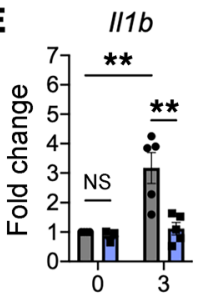

Days after IRI

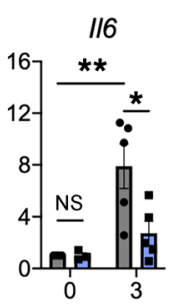

Days after IR

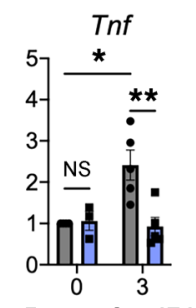

Days after IRI

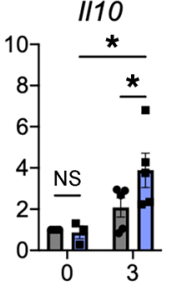

Days after IR

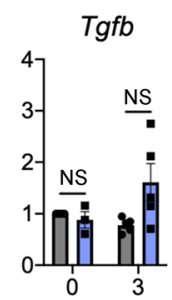

Days after IR
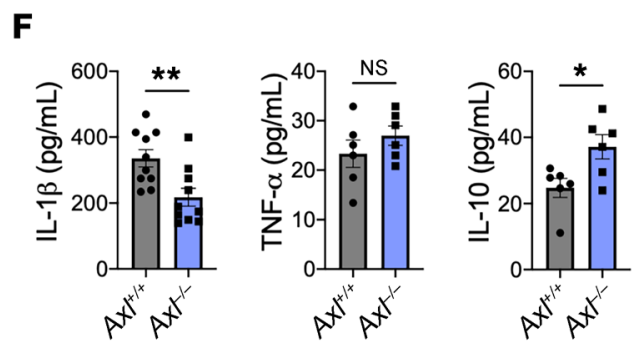

Figure 3. Macrophage AXL promotes inflammatory responses after myocardial ischemia/reperfusion infarction (IRI). Total number of (A) neutrophils and (B) Ly6 $C^{h i}$ monocytes within the infarcted myocardium as measured on the days indicated after IRI in $A x^{I^{+/+}}$or $A x^{l^{-/}}$mice. Flow plots depict events 3 days after IRI. (C) Total number of MerTK+ macrophages within the infarcted myocardium as measured on the indicated days after IRI in $A X^{l^{+/}}$or $A x^{l^{-/-}}$

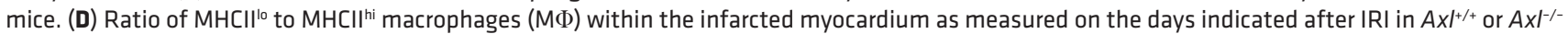
mice. For A-D, $n=4-5$ mice/group pooled from 3 independent experiments. ${ }^{*} P<0.05,{ }^{*} P<0.01$ by 2 -way ANOVA followed by Tukey's test. (E) Gene expression of pro- and antiinflammatory mediators in whole-infarct extracts from $A x l^{+/+}$or $A x l^{-/-}$mice. $n=3-5$ mice/group pooled from 3 independent experiments. ${ }^{*} P<0.05$, ${ }^{* *} P<0.01$ by 2 -way ANOVA followed by Tukey's test. (F) Serum levels of pro- and antiinflammatory mediators as measured 3 days after IRI from in $A x I^{+/+}$or $A x l^{-/-}$mice. $n=6-10$ mice/group pooled from 3 independent experiments. ${ }^{*} P<0.05$, ${ }^{* *} P<0.01$ by 2-tailed, unpaired $t$ test. All data presented as mean $\pm \mathrm{SEM}$.

increased TLR4 priming of the inflammasome as measured by increased $I l 1 b$ expression in $A x l^{+/+} \mathrm{BMDMs}$, but these effects were absent from $\mathrm{Axt}^{--}$BMDMs (Figure 4C). Furthermore, inflammasome activation with ATP was also impaired by $A x l$ deficiency as measured by a reduction in both activated caspase-1 (Figure 4, $D$ and E) and secretion of IL-1 $\beta$ into the culture media (Figure $4 F$ ), supporting the concept that AXL signaling augments TLR4 priming of the NLRP3 inflammasome. Impaired inflammatory activation in $\mathrm{Axt}^{-/}$BMDMs was also evidenced by a decrease in TNF- $\alpha$ levels with a concomitant increase in IL-10 levels after TLR4 stimulation (Figure 4, $\mathrm{G}$ and $\mathrm{H}$ ).

Macrophages activated by TLR4 signaling require a switch to glycolytic metabolism to facilitate the increased energetic demands necessary to produce IL-1 $\beta$ (23). To determine whether
AXL signaling was required for inflammatory glycolytic reprogramming, we measured the extracellular acidification rate of $A x l^{+++}$and $A x^{-/}$BMDMs after TLR4 stimulation. Although we observed an increase in glycolysis in $\mathrm{Axl}^{+/+} \mathrm{BMDMs}$ after TLR4 stimulation, this effect was largely absent in $A x t^{-1}$ BMDMs (Figure $5 \mathrm{~A}$ ). AXL signaling alone, using an AXL-agonizing antibody, was also sufficient to promote an increase in glycolysis compared with an isotype control, albeit to a lesser extent than TLR4 stimulation (Figure 5B). To test whether AXL and TLR4 can be in spatial proximity to promote inflammatory glycolytic reprogramming, we performed a proximity ligation assay. Although the assay revealed data consistent with a close interaction between AXL and TLR4 in BMDMs (Supplemental Figure 8A), no differences in NF- $\mathrm{kB}$ signaling were observed between $A x^{+/+}$and $A x l^{-/}$BMDMs after TLR4 stimulation 
A
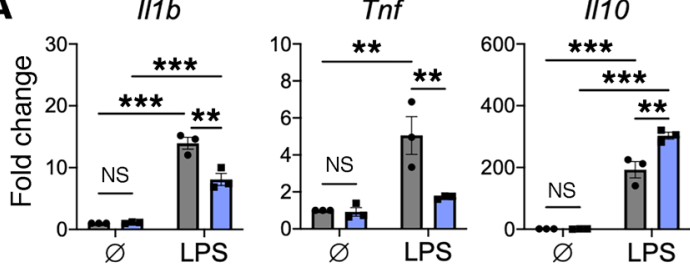

B

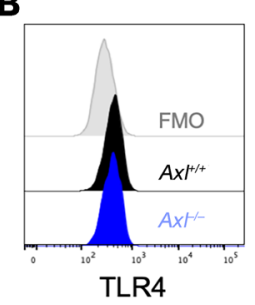

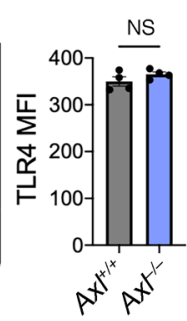
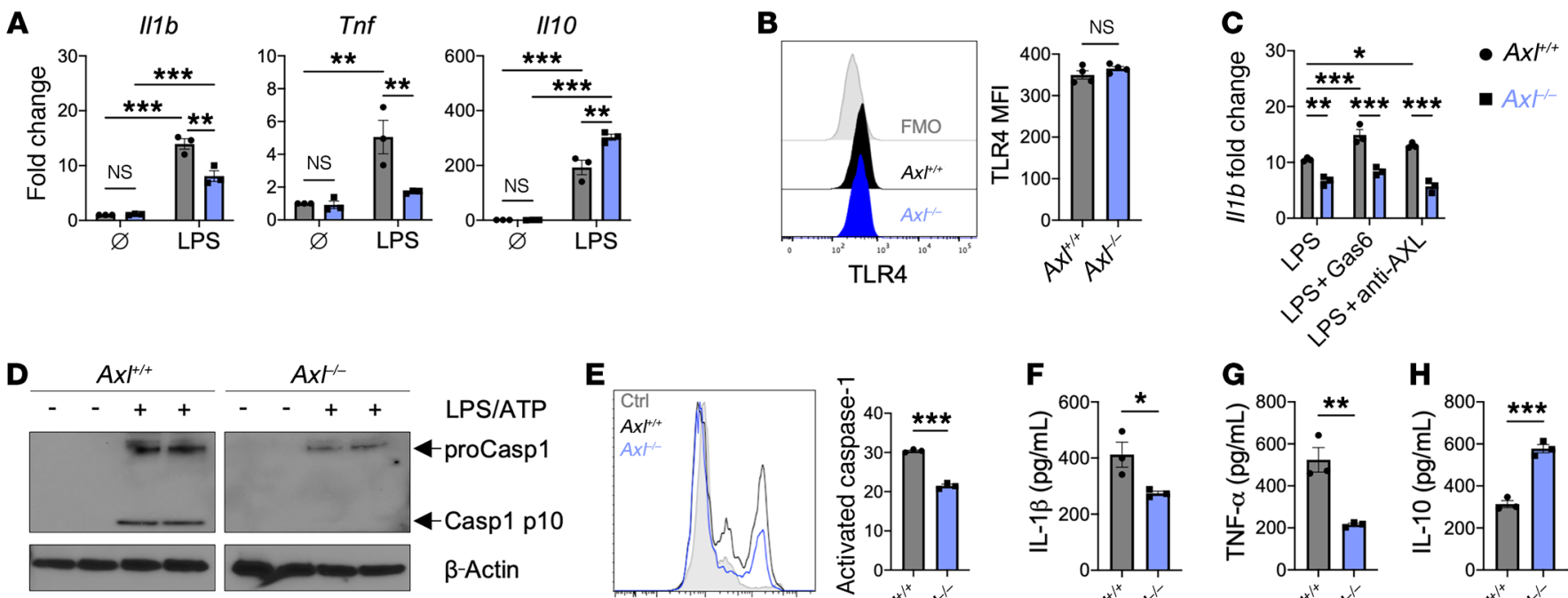

$\mathbf{E}$
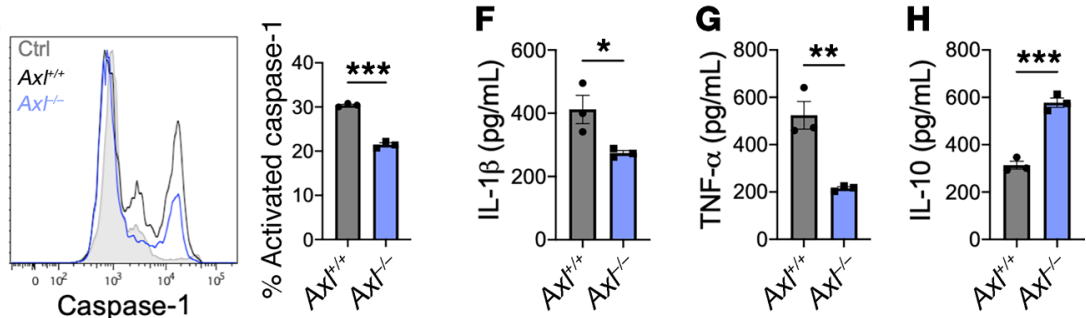

Figure 4. AXL signaling augments TLR4 priming of the inflammasome in macrophages. (A) Pro- and antiinflammatory gene expression in $A x l^{+/+}$or $\mathrm{Ax}^{-1-}$ bone marrow-derived macrophages (BMDMs) untreated (Ø) or treated with lipopolysaccharide (LPS) for 6 hours. Data are representative of more than 3 independent experiments. $n=3$ sets of cells/group. ${ }^{* *} P<0.01,{ }^{* *} P<0.001$ by 2 -way ANOVA followed by Tukey's test. (B) TLR4 expression on untreated $A x l^{+/+}$or $A x l^{-/-}$BMDMs as measured by flow cytometry. Data are representative of 2 independent experiments. $n=4$ sets of cells/group. NS, not significant by 2-tailed, unpaired $t$ test. (C) Gene expression of $1 / 1 \mathrm{~b}$ in serum-starved $A x^{1^{+/}}$or $A x^{1 /-}$ BMDMs treated with LPS and AXL-specific agonists, growth arrest-specific 6 (Gas6) protein, or anti-mouse AXL agonizing antibody, for 6 hours. Data are representative of 3 independent experiments. $n=3$ sets of cells/group. ${ }^{*} P<0.05,{ }^{*} P<0.01,{ }^{* *} P<0.001$ by 2 -way ANOVA followed by Tukey's test. (D) Immunoblot or (E) flow cytometry of caspase-1 activation in $A x^{+/+}$or Axl ${ }^{-/-}$BMDMs primed with LPS for 3 hours followed by activation with ATP for 30 minutes. Data are representative of 3 independent experiments. $n=2-3$ sets of cells/group. ${ }^{* *} P<0.001$ by 2 -tailed, unpaired $t$ test. See complete unedited blots in the supplemental material. (F) IL-1 $\beta$ production by $A x^{+/+}$or $A x l^{1 /-}$ BMDMs primed with LPS for 3 hours followed by activation with ATP for 30 minutes. (G) TNF- $\alpha$ or (H) IL-10 production by $A \mathrm{Xl}^{+/+}$or $A \mathrm{Xl}^{-/-}$BMDMs treated with LPS for 6 hours. For $\mathbf{F}-\mathbf{H}$, data are representative of more than 3 independent experiments. $n=3$ sets of cells/group. ${ }^{*} P<0.05,{ }^{* *} P<0.01,{ }^{* *} P<0.001$ by 2 -tailed, unpaired $t$ test. All data presented as mean \pm SEM.

(Supplemental Figure 8B), suggesting that AXL augmented glycolysis independently of NF- $\mathrm{KB}$ activation. Hypoxia-inducible factor $1 \alpha$ (HIF-1 $\alpha$ ) has been implicated in the TLR4-mediated switch to glycolytic metabolism (24), so we next investigated whether $A x l$ deficiency impacted HIF-1 $\alpha$ activation. Relative to $A x l^{t^{+/}}$BMDMs, there were reductions in both gene and protein expression of HIF$1 \alpha$ in $A x t^{--}$BMDMs (Figure 5, C, E, and F), which was associated with a decrease in HIF-1 $\alpha$-dependent genes involved in glycolytic metabolism (Figure 5D). Treatment of BMDMs with cobalt chloride, a pharmacological HIF activator, restored glycolytic metabolism in $A x^{--}$BMDMs to a level similar to that of $A x^{+++}$BMDMs (Figure 5G), suggesting that AXL signaling is required for HIF-1 $\alpha-$ dependent glycolytic proinflammatory reprogramming of macrophages in response to inflammatory stimuli.

AXL signaling promotes phosphorylation and activation of STAT1 $(7,18)$, which has been separately linked to both HIF-1 $\alpha$ signaling (25) and IL-1 $\beta$ production (26). To test whether AXL was required for STAT1 signaling after TLR4 stimulation, we measured phosphorylation of STAT1 (p-STAT1, Tyr701) in BMDMs after TLR4 stimulation or in cardiac macrophages after IRI. Treatment of $A \mathrm{xl}^{+/+} \mathrm{BMDMs}$ with the AXL-specific agonist Gas6 revealed that while Gas6/AXL signaling alone was sufficient to induce p-STAT1, it was insufficient to increase glycolytic metabolism or IL-1 $1 \beta$ production (Supplemental Figure 9). TLR4 stimulation also increased p-STAT1 levels in $\mathrm{Axl}^{+++}$BMDMs (Figure 6, A and B) and the addition of exogenous Gas 6 augmented both p-STAT1 levels and IL-1 $\beta$ production during TLR4 stimulation (Supplemental Figure 9). In contrast, p-STAT1 levels were attenuated in Axt ${ }^{--}$BMDMs after TLR4 stimulation (Figure 6, A and B). Similarly, p-STAT1 levels were reduced after IRI in cardiac macrophages from $A x t^{--}$mice compared with $A x^{l^{+/}}$mice (Figure 6C), supporting a crucial role for AXL in STAT1 activation. To test whether STAT1 signaling was required for TLR4-mediated glycolytic proinflammatory reprogramming of macrophages, we measured metabolic and inflammatory function in $\mathrm{Stat1}^{+/+}$and Stat1-- BMDMs after TLR4 stimulation. In contrast to Stat1 $^{+/+}$BMDMs, HIF-1 $\alpha$ activation was attenuated in Stat1 ${ }^{-/}$BMDMs after TLR4 stimulation (Figure 6D), which was associated with an impaired switch to glycolytic metabolism (Figure $6 \mathrm{E}$ ) and reduced secretion of IL-1 $\beta$ (Figure $6 \mathrm{~F}$ ). Taken together, these results support a role for Gas6/AXL signaling in augmenting TLR4 activation of HIF-1 $\alpha$ through STAT1 signaling to promote the glycolytic reprogramming to inflammatory macrophages.

As cardiac macrophages express AXL and MerTK, both of which are efferocytosis receptors, one hypothesis is that in addition to impairing proinflammatory macrophage reprogramming, loss of AXL would improve cardiac repair through increased cardioprotective MerTK-dependent phagocytic clearance of apoptotic cardiomyocytes. To test whether enhanced efferocytosis restrained inflammation and improved cardiac repair in $\mathrm{Axl}^{-/-}$mice, we quantified post-IRI association of fluorescent and cardiomyocyte-specific mCherry protein with cardiac macrophages. While IRI led to significantly increased macrophage clearance of cardiomyocyte debris, no difference in reperfusion-dependent efferocytosis of dead cardiomyocytes 

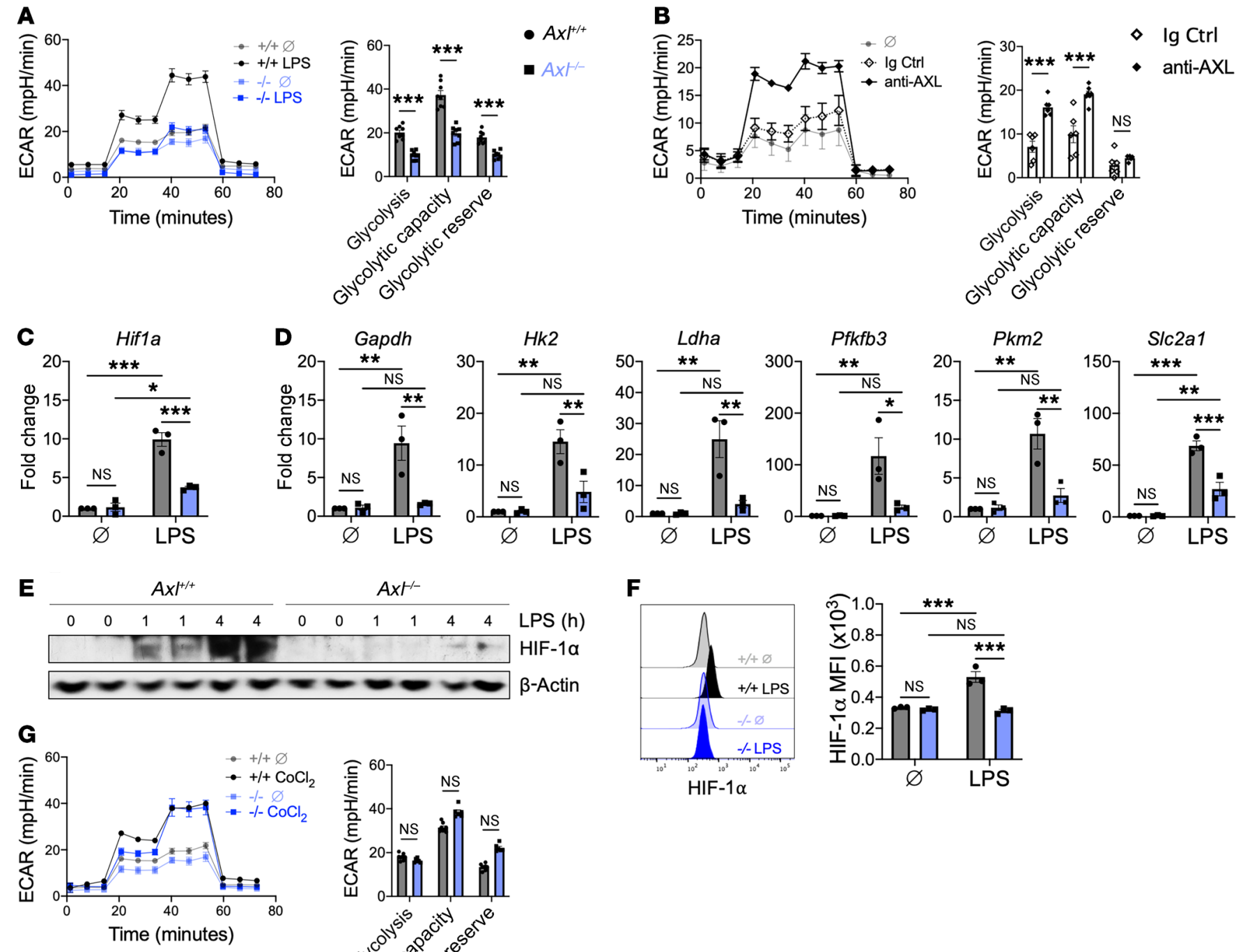

$\mathrm{Axt}^{--}$
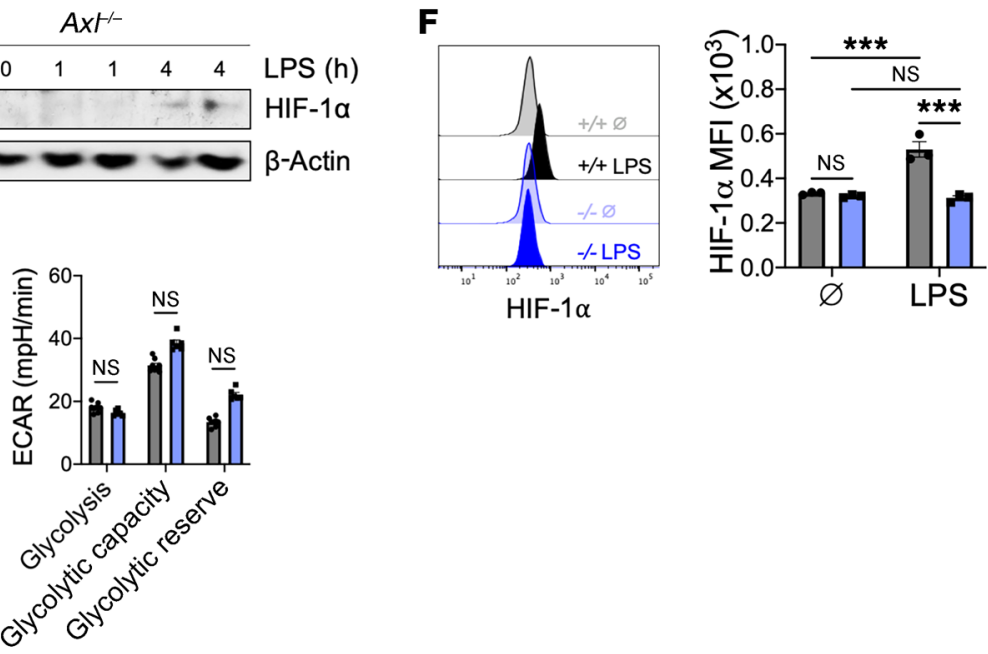

Figure 5. AXL signaling is required for the switch to glycolytic metabolism to fuel proinflammatory responses in macrophages. (A) Extracellular acidification rate (ECAR) in $A x^{+/+}$or $A x l^{-/-}$bone marrow-derived macrophages (BMDMs) untreated (+/+ $\emptyset$ and -/- $\emptyset$ ) or treated with lipopolysaccharide (LPS) for 3 hours, with quantification of glycolytic function. (B) ECAR with quantification of glycolytic function in $A x^{l^{++}}$BMDMs treated with anti-mouse AXL agonizing antibody or isotype control. For $\mathbf{A}$ and $\mathbf{B}$, data are representative of 2-3 independent experiments. $n=5$-8 sets of cells/group. ${ }^{* * *} P<0.001$ by 2-tailed, unpaired $t$ test. (C) Hif1a gene expression in BMDMs treated with LPS for 3 hours. (D) Expression of genes involved in glycolytic metabolism in BMDMs treated with LPS for 3 hours. For $\mathbf{C}$ and $\mathbf{D}$, data are representative of 2 independent experiments. $n=3$ sets of cells/group. ${ }^{*} P<0.05,{ }^{*} P<0.01,{ }^{* * *} P<0.001$ by 2 -way ANOVA followed by Tukey's test. (E) Immunoblot or (F) flow cytometry of HIF-1 $\alpha$ protein in BMDMs treated with LPS. Data are representative of 2 independent experiments. $n=3$ sets of cells/group. ${ }^{* *} P<0.001$ by 2 -way ANOVA followed by Tukey's test. See complete unedited blots in the supplemental material. (C) ECAR with quantification of glycolytic function measured in $A x^{1 /+}$ or $A x^{1--}$ BMDMs treated with the HIF-1 $\alpha$ pharmacological activator, cobalt chloride $\left(\mathrm{CoCl}_{2}\right)$. Untreated groups $(+/+\emptyset$ and $-/-\emptyset)$ are the same as presented in panel A. Data are representative of 2 independent experiments. $n=6-8$ sets of cells/group. NS, not significant by 2 -tailed, unpaired $t$ test. All data presented as mean \pm SEM.

was observed between $A x l^{+/+}$and $A x l^{l^{--}}$mice (Figure 7A). The phagocytosis-independent cardioprotection observed during Axl deficiency suggests that AXL and MerTK function independently to regulate reprogramming of macrophages.

We next tested whether AXL and MerTK exhibited divergent and independent function after IRI by comparing LV remodeling and contractile function in mice deficient in either receptor alone or deficient in both AXL and MerTK. In contrast to loss of AXL alone, loss of MerTK alone worsened cardiac repair and contractile function after IRI (Figure 7B), as previously reported by us and others $(11,12)$. Importantly, loss of either AXL or MerTK did not result in a compensatory increase in the other receptor on cardiac macrophages (Figure 7C). Surprisingly, mice deficient in both $A x l$ and Mertk exhibited a cardioprotective response after IRI that was similar to that of mice deficient in Axl alone, with improvements in cardiac repair and preservation of systolic function compared with controls or mice deficient in Mertk alone (Figure 7, B and D). Flow cytometric analyses 
A

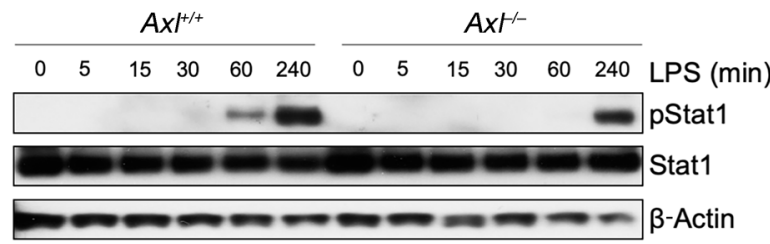

C

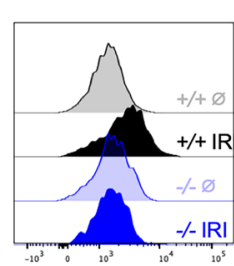

PSTAT1

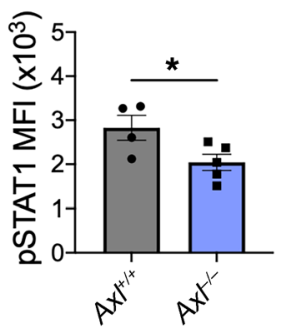

B
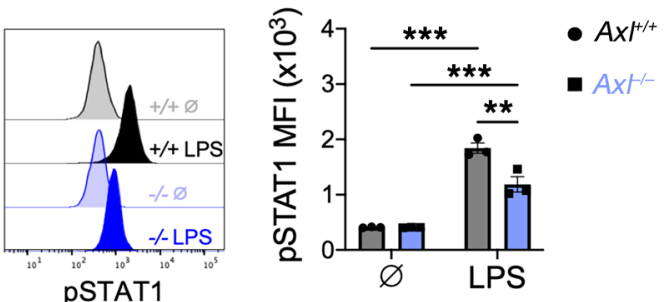

D pSTAT1
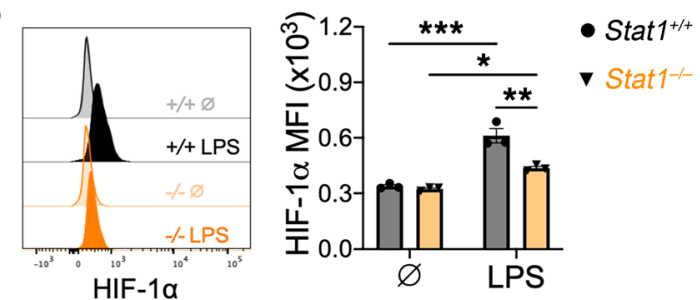

E
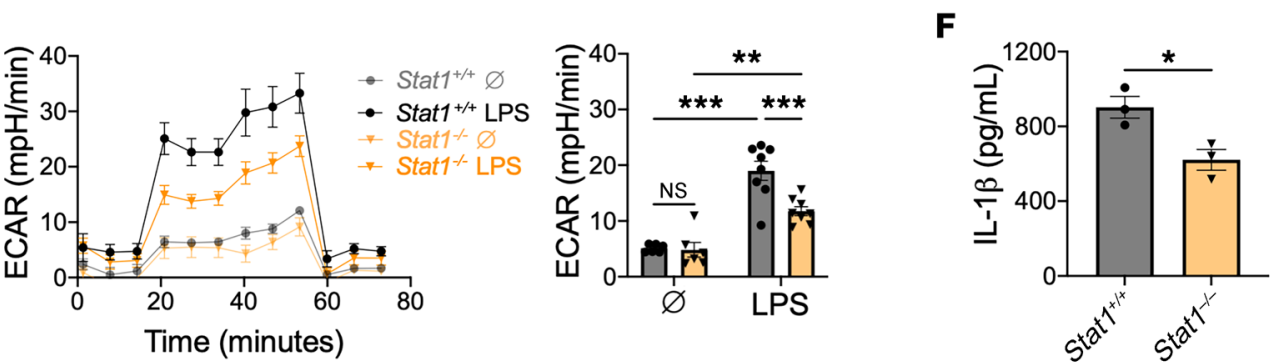

Figure 6. Cross signaling between AXL and TLR4 augments STAT1 activation to fuel proinflammatory responses in macrophages. (A) Immunoblot or (B) flow cytometry of STAT1 phosphorylation in $\mathrm{Axl}^{+/+}$or $A \mathrm{xl}^{-/-}$bone marrow-derived macrophages (BMDMs) untreated (Ø) or treated with lipopolysaccharide (LPS). Data are representative of $2-3$ independent experiments. $n=3$ sets of cells/group. ${ }^{* *} P<0.01,{ }^{* * *} P<0.001$ by 2 -way ANOVA followed by Tukey's test. See complete unedited blots in the supplemental material. (C) STAT1 phosphorylation in $A x^{I^{+/+}}$or $A x^{1--}$ cardiac macrophages 3 days after ischemia/ reperfusion infarction (IRI). $n=4-5$ mice/group pooled from 2 independent experiments. ${ }^{*} P<0.05$ by 2-tailed, unpaired $t$ test. (D) HIF-1 $\alpha$ protein in Stat $1^{+/+}$ or Stat $1^{-1-}$ BMDMs treated with LPS for 4 hours as measured by flow cytometry. Data are representative of 2 independent experiments. $n=3$ sets of cells / group. ${ }^{*} P<0.05$, ${ }^{*} P<0.01,{ }^{* *} P<0.001$ by 2 -way ANOVA followed by Tukey's test. (E) Extracellular acidification rate (ECAR) with quantification of glycolytic function in Stat ${ }^{+/+}$or Stat1 ${ }^{-/-}$BMDMs treated with LPS for 3 hours. Data are representative of 2 independent experiments. $n=6-8$ sets of cells/ group. ${ }^{*} P<0.05$, ${ }^{* * *} P<0.001$ by 2 -way ANOVA followed by Tukey's test. (F) IL-1 $\beta$ production by Stat1 $1^{+/+}$or Stat1 ${ }^{-/-}$BMDMs primed with LPS for 3 hours followed by activation with ATP for 30 minutes. Data are representative of 2 independent experiments. $n=3$ sets of cells/group. ${ }^{*} P<0.05$ by 2 -tailed, unpaired $t$ test. All data presented as mean \pm SEM.

revealed an increase in reparative $\mathrm{MHCII}{ }^{\mathrm{lo}}$ cardiac macrophages in $\mathrm{Axl}^{-/-} \mathrm{Mertk}^{-/}$mice compared with $\mathrm{Axl}^{+/+} \mathrm{Mertk}^{+/+}$mice after IRI (Figure 7E), suggesting that AXL favors accumulation of proinflammatory $\mathrm{MHCII}^{\mathrm{hi}}$ macrophages to worsen cardiac repair after IRI. Taken together, these results support divergent roles for AXL and MerTK in mediating pathogenic and protective macrophage responses, respectively, after IRI.

To test the therapeutic potential of these findings, we treated mice daily after IRI with a small molecule inhibitor of AXL, R428, that binds specifically $\left(\mathrm{IC}_{50}=14 \mathrm{nM}\right)$ and with high selectivity to the AXL intracellular catalytic kinase domain and inhibits its activity (50-fold relative to MerTK, ref. 27). Treatment of WT mice with the AXL inhibitor significantly reduced infarct size, demonstrating that transient inhibition of AXL confers cardioprotection after IRI (Figure 7F). Similar cardioprotection was observed following administration of AXL inhibitor to $\mathrm{Mertk}^{-/}$mice after IRI (Figure 7G), consistent with independent functions for AXL and MerTK in the infarcted ventricle. We have previously shown that the beneficial effects of MerTK are compromised by its proteolytic cleavage after IRI and that strategies that block reperfusion-associated
MerTK cleavage improve cardiac function (12). To test whether targeting both AXL and MerTK could synergize to enhance cardiac repair after IRI, we treated mice with a cleavage-resistant MerTK (Mertk ${ }^{C R / C R}$, which preserves the cardioprotective effects naturally compromised after IRI, with the AXL-specific inhibitor. Treatment of Mertk ${ }^{C R / C R}$ mice with AXL inhibitor reduced infarct size compared with either intervention alone (Figure 7F), demonstrating that strategies that combine inhibition of AXL signaling and MerTK cleavage may confer the greatest preservation of cardiac function after IRI.

Like MerTK, AXL is also proteolytically cleaved to release a stable cleavage product called solAXL. In ST-segment elevation MI (STEMI) patients, solAXL levels are increased during the acute phase and are associated with adverse ventricular remodeling and progression to heart failure (14). We independently validated this finding in a small cohort of STEMI patients and observed a similar increase in serum levels of solAXL in mice after IRI (Supplemental Figure 10, A and B). To test the role of AXL cleavage after IRI, we generated gene-targeted mice $\left(A x l^{C R / C R}\right)$ in which the proteolytic site of AXL was deleted, leading to replacement of the WT AXL with 
A

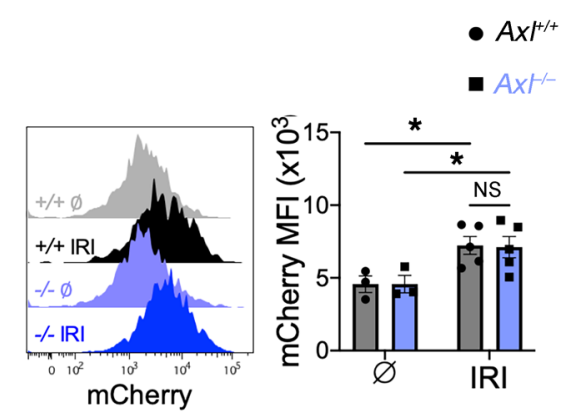

C
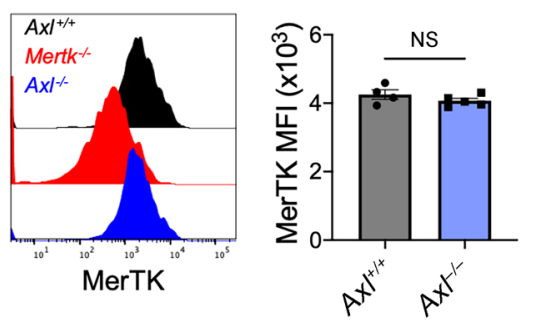

$\mathbf{E}$
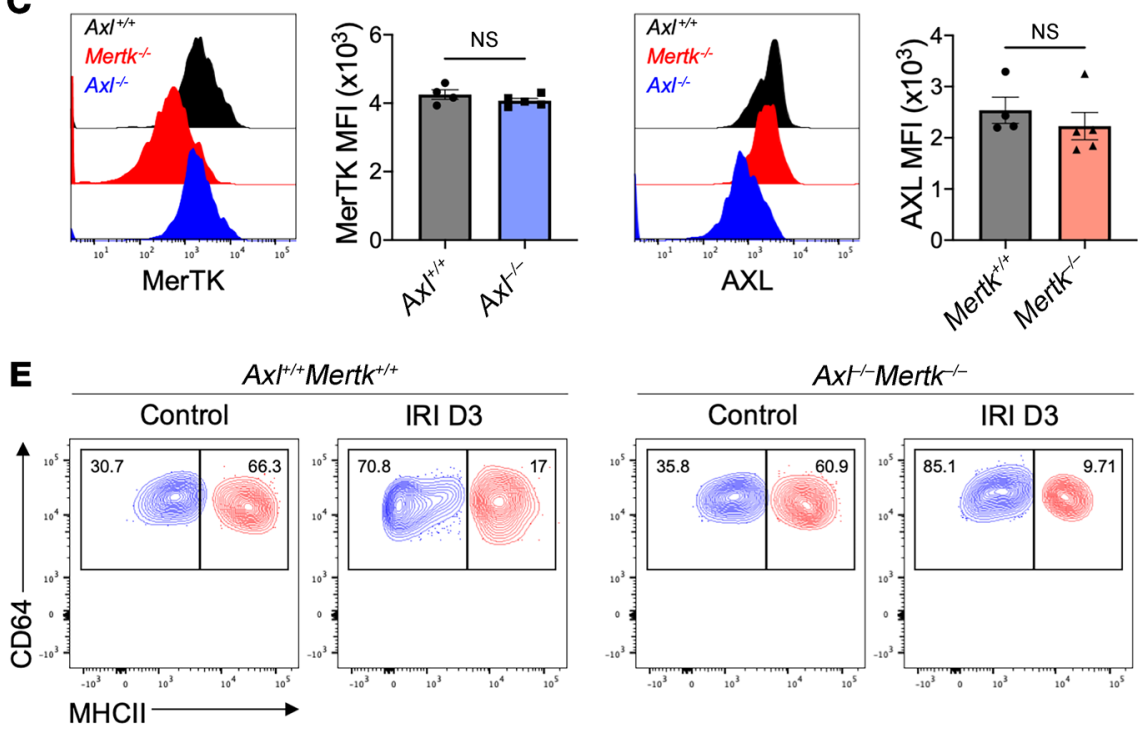
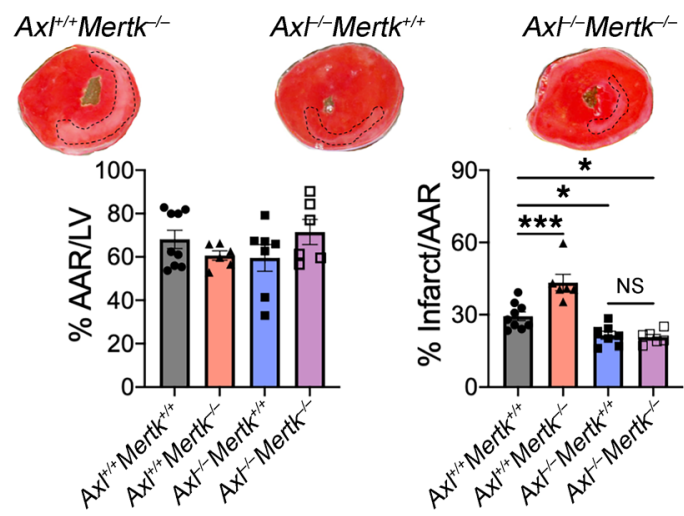

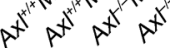

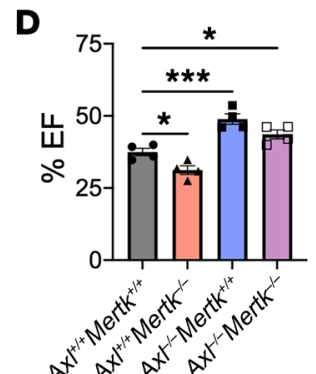

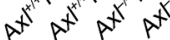

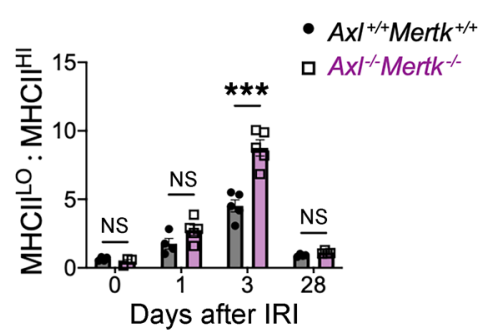

$\mathbf{F}$
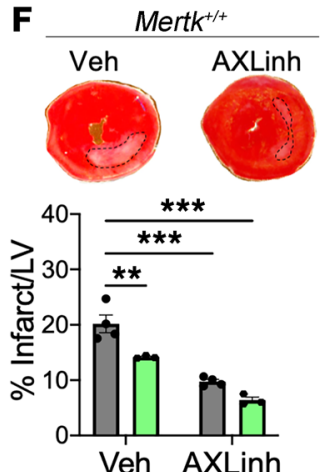
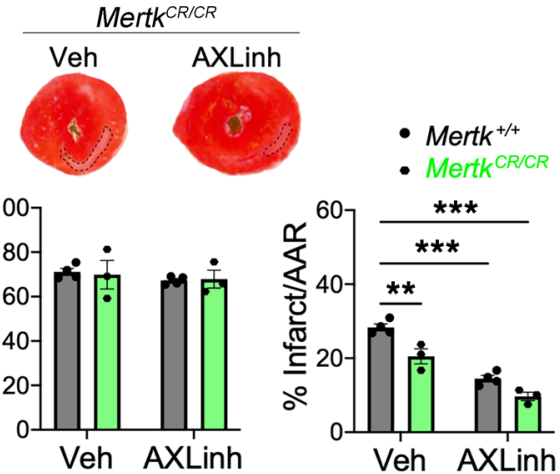

G

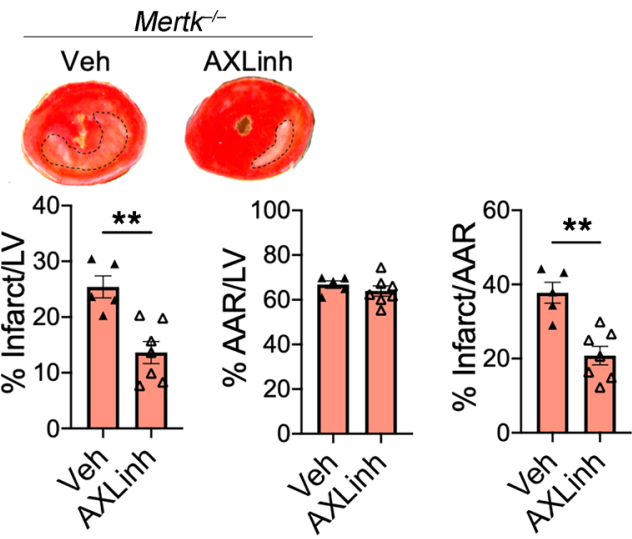

Figure 7. Divergent roles for AXL and MerTK in cardiac repair after myocardial ischemia/reperfusion infarction (IRI). (A) Phagocytosis of apoptotic mCherry-expressing cardiomyocytes by cardiac macrophages from $A x^{l^{+/+}}$or $A x^{l^{-1-}}$ mice at baseline ( $\emptyset$ ) or 4 hours after IRI. $n=3-5$ mice/group pooled from 3 independent experiments. ${ }^{*} P<0.05$ by 2-way ANOVA followed by Tukey's test. (B) Percentage infarct/left ventricle (LV), percentage area at risk (AAR)/

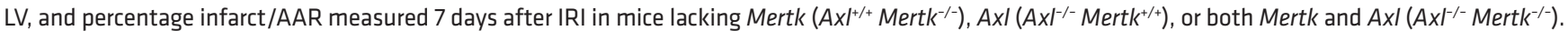
$n=6-9$ mice/group pooled from more than 3 independent experiments. ${ }^{*} P<0.05$, ${ }^{*} P<0.01$, ${ }^{* * *} P<0.001$ by 1 -way ANOVA followed by Tukey's test. (C) Expression of MerTK and AXL on cardiac macrophages from Axl-or Mertk-deficient mice as measured 3 days after IRI. $n=4-5$ mice/group pooled from 2 independent experiments. NS, not significant by 2-tailed, unpaired $t$ test. (D) Quantification of percentage ejection fraction (\% EF) in mice 28 days after IRI $n=4$ mice/group pooled from 4 independent experiments. ${ }^{*} P<0.05,{ }^{* * *} P<0.001$ by 1 -way ANOVA followed by Tukey's test. (E) Ratio of MHCII' to MHCII ${ }^{\text {hi }}$ cardiac macrophages within the infarcted myocardium of $\mathrm{Axl}^{l^{++}}$Mertk ${ }^{+/+}$or $A x \mathrm{I}^{1--}$ Mertk $\mathrm{k}^{-1-}$ mice. $n=3-5$ mice/group pooled from 3 independent experiments. ${ }^{* * *} P<0.001$ by 2 -way ANOVA followed by Tukey's test. (F) Infarct measurements 7 days after IRI in Mertk ${ }^{+/+}$or Mertk ${ }^{C R / C R}$ mice treated with the AXL-selective inhibitor R428 or vehicle. $n=3-4$ mice/group pooled from 3 independent experiments. ${ }^{* *} P<0.01,{ }^{* *} P<0.001$ by 2 -way ANOVA followed by Tukey's test. (G) Infarct measurements 7 days after IRI in Mertk ${ }^{-1-}$ mice treated with the AXL-selective inhibitor R428 or vehicle. $n=5-7$ mice/group pooled from 3 independent experiments. ${ }^{*} P<0.01$ by 2 -tailed, unpaired $t$ test. All data presented as mean \pm SEM. 
A

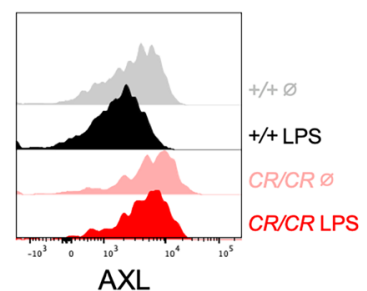

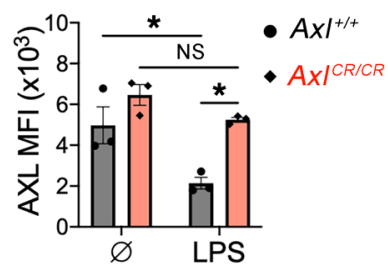
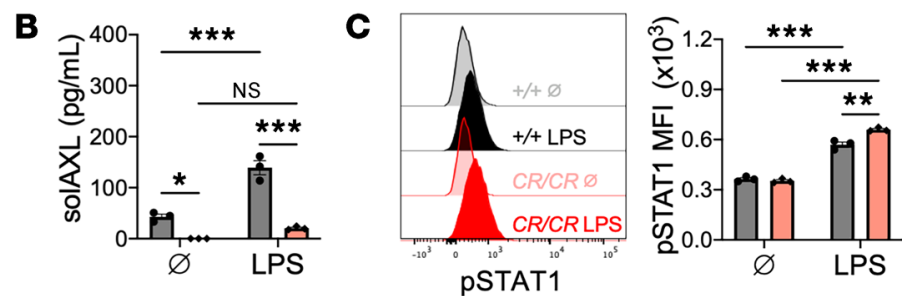

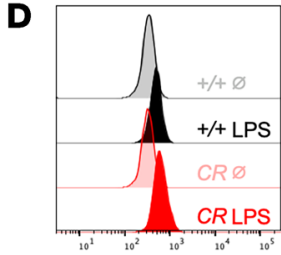

HIF-1 $\alpha$

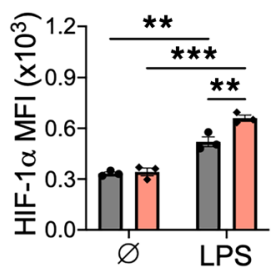

LPS
E

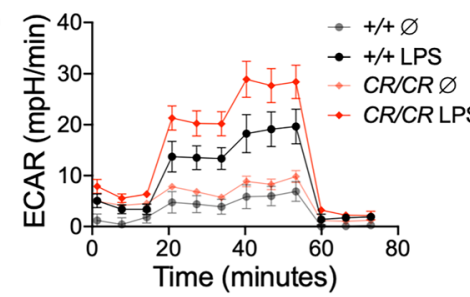

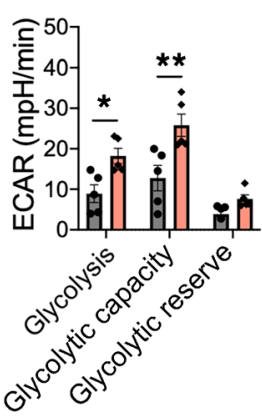

$\mathbf{F}$

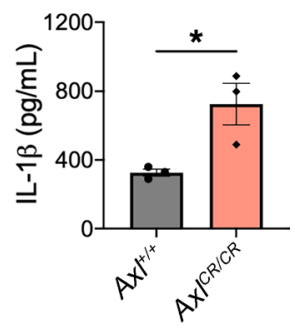

Figure 8. AXL cleavage limits proinflammatory responses in macrophages. (A) Cell-surface expression of AXL on bone marrow-derived macrophages (BMDMs) from wild-type $\left(A x l^{++}\right)$or $A X L$ cleavage-resistant $\left(A x I^{C R / C R}\right)$ mice untreated $(\varnothing)$ or treated with lipopolysaccharide (LPS) for 1 hour. (B) Soluble $A X L$ (solAXL) in culture media of BMDMs treated with LPS for 1 hour. (C) STAT1 phosphorylation in BMDMs treated with LPS for 1 hour. (D) HIF-1 $\alpha$ protein in BMDMs treated with LPS for 4 hours. For A-D, data are representative of 3 independent experiments. $n=3$ sets of cells/group. ${ }^{*} P<0.05,{ }^{* *} P<0.01,{ }^{* * *} P<$ 0.001 by 2 -way ANOVA followed by Tukey's test. (E) Extracellular acidification rate (ECAR) with quantification of glycolytic function in BMDMs treated with LPS for 3 hours. Data are representative of 2 independent experiments. $n=5$ sets of cells/group. ${ }^{*} P<0.05,{ }^{* *} P<0.01$ by 2 -tailed, unpaired $t$ test. (F) IL-1 $\beta$ production by BMDMs treated with LPS for 18 hours. $n=3$ sets of cells/group. ${ }^{*} P<0.05$ by 2 -tailed, unpaired $t$ test. All data presented as mean \pm SEM.

a fully functional but cleavage-resistant AXL (Supplemental Figure $11, \mathrm{~A}-\mathrm{D})$. To assess cleavage resistance, BMDMs from $A x l^{C R / C R}$ and $A x l^{+++}$mice were treated with a TLR4 agonist and then assayed for cell-surface AXL and solAXL by flow cytometry, ELISA, and immunoblot. TLR4 agonism decreased cell-surface AXL and increased solAXL in $\mathrm{Axl}^{+++}$macrophages, but these effects were largely absent with $A x l^{C R / C R}$ macrophages (Figure 8, A and B, and Supplemental Figure 11E). Consistent with a role for AXL in the proinflammatory metabolic reprogramming of macrophages, we observed increased p-STAT1 and HIF-1 $\alpha$ signaling in Axl ${ }^{C R / C R}$ BMDMs compared with $A x l^{+++}$BMDMs (Figure 8, C and D), which was associated with elevated glycolysis and IL-1 $\beta$ secretion in response to TLR4 stimulation (Figure 8, E and F). In $A x l^{C R / C R}$ mice, cell-surface AXL was preserved on cardiac macrophages after IRI (Figure 9A) and serum solAXL was significantly reduced at both steady state and after IRI compared with $A x l^{+/+}$mice (Figure 9B). In contrast to the increase in solAXL levels observed in $A x^{l^{++}}$mice after IRI, $A x l^{C R / C R}$ mice exhibited no change in solAXL levels after IRI, validating the $A x^{C R /}$ ${ }^{C R}$ mouse as a model for testing the functional consequences of AXL cleavage in vivo. To determine the functional role of AXL cleavage in the acute phase after IRI, we compared inflammation and tissue repair between $A x l^{+/+}$and $A x l^{C R / C R}$ mice after IRI. Relative to $A x l^{+/+}$ mice, infarct sizes were increased in $A x^{C R / C R}$ mice 7 days after IRI (Figure 9C). The adverse ventricular remodeling in $A x^{C R / C R}$ mice was associated with an increased inflammatory response, as measured by increased neutrophils (Figure 9D), Ly6C $\mathrm{C}^{\text {hi }}$ monocytes (Figure 9E), and inflammatory MHCII ${ }^{\text {hi }}$ macrophages (Figure 9F) within the infarct and elevated serum levels of IL-1B (Figure 9G) compared with $\mathrm{Axl}^{+++}$mice, revealing that AXL cleavage limits adverse ventricular remodeling after IRI.

\section{Discussion}

Our data reveal a role for AXL in adverse cardiac remodeling after IRI. According to our working model (Supplemental Figure 12), release of DAMPs secondary to IRI promotes increased AXL levels on cardiac macrophages. Cross signaling between AXL and TLR4 augments STAT1 signaling to direct a HIF-1 $\alpha$-dependent switch to glycolytic metabolism in cardiac macrophages and secretion of proinflammatory IL-1 $\beta$. This leads to increased intramyocardial inflammation and culminates in adverse ventricular remodeling and impaired contractile function. AXL functions independently of cardioprotective MerTK to worsen cardiac repair, but like MerTK, is proteolytically cleaved. Finally, administration of a selective small molecule inhibitor of AXL alone reduced cardiac damage. AXL inhibition in combination with blockade of MerTK cleavage further improved cardiac healing after IRI. This interesting AXL-MerTK dichotomy strengthens the foundation to investigate macrophage TAM receptors as targets for cardioprotection in the infarcted human heart.

Although a proinflammatory role for AXL in cardiac macrophages was somewhat surprising, given its well-characterized role in efferocytosis (28), emerging evidence from both humans and experimental models is beginning to support a detrimental role for AXL in a variety of diseases $(29,30)$. In our model of clinically relevant myocardial IRI, we observed both increased serum levels of solAXL and cell-surface AXL expression on cardiac macrophages, the latter of which promoted proinflammatory IL-1 $\beta$ production. Using a newly generated cleavage-resistant AXL mouse, we discovered that the increase in solAXL by proteolytic cleavage of cell-surface AXL functioned to limit proinflammatory macrophage responses in vitro and adverse 
A
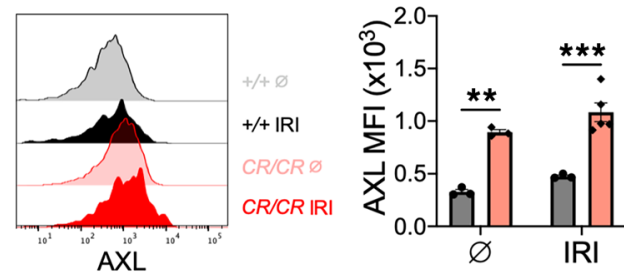

C
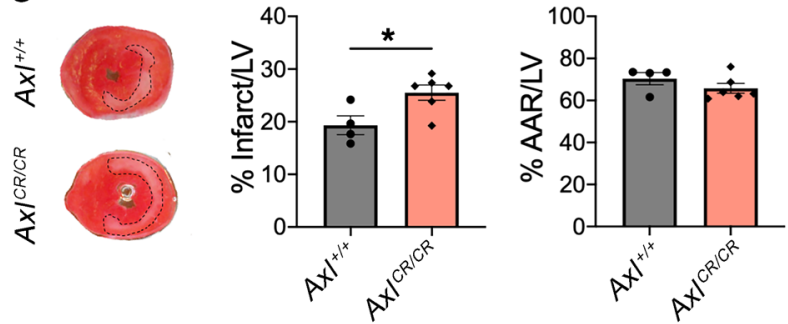

D
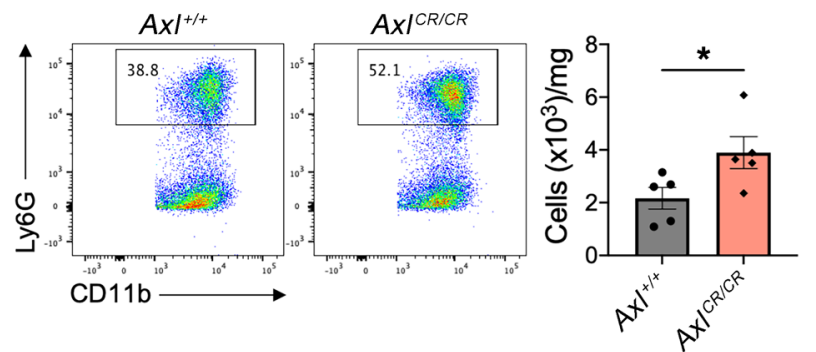

$\mathbf{F}$

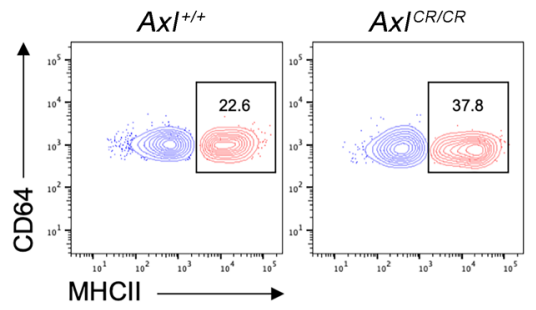

B
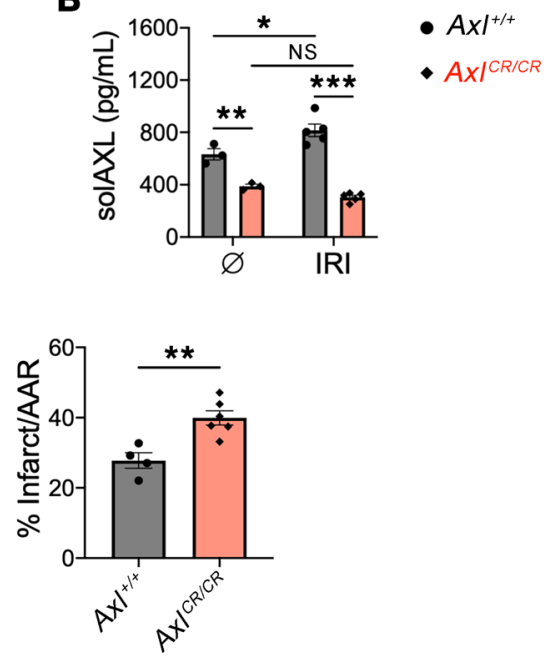

E
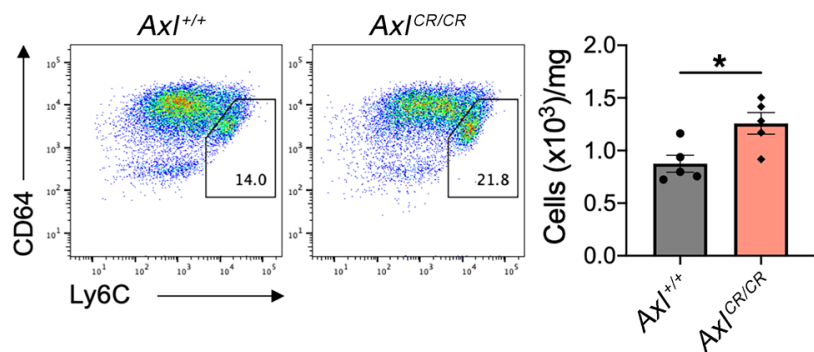

G

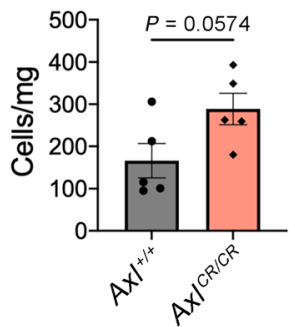

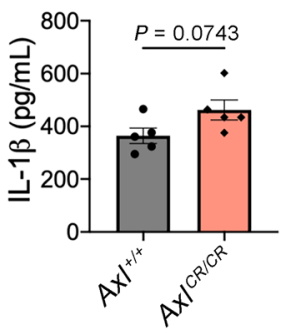

Figure 9. AXL cleavage limits adverse ventricular remodeling after myocardial ischemia/reperfusion infarction (IRI). (A) Cell-surface expression of AXL on cardiac macrophages from wild-type $\left(A x l^{++}\right)$or $A X L$ cleavage-resistant $\left(A x I^{[R / C R}\right)$ mice before (Ø) or 3 days after IRI. (B) Serum levels of soluble AXL (solAXL) before or 3 days after IRI. For $\mathbf{A}$ and $\mathbf{B}, n=3-5$ mice/group pooled from 3 independent experiments. ${ }^{*} P<0.05,{ }^{* *} P<0.01,{ }^{* * *} P<0.001$ by 2-way ANOVA followed by Tukey's test. (C) Percentage infarct/left ventricle (LV), percentage area at risk (AAR)/LV, and percentage infarct/AAR measured 7 days after IRI in $\left.A x\right|^{+++}$or $A x{ }^{[R / C R}$ mice. $n=4-6$ mice/group pooled from 3 independent experiments. ${ }^{*} P<0.05$, ${ }^{* *} P<0.01$ by 2 -tailed, unpaired $t$ test. Total number of (D) neutrophils, (E) Ly6C hi monocytes, and (F) MHCII hi macrophages within the infarct 3 days after IRI. Flow plots depict events 3 days after IRI. (G) Serum levels of IL-1 $\beta$ as measured 3 days after IRI. For $\mathbf{D}-\mathbf{G}, n=5$ mice/group pooled from 2 independent experiments. ${ }^{*} P<0.05$ by 2 -tailed, unpaired $t$ test. All data presented as mean \pm SEM.

ventricular remodeling during the acute phase after IRI. AXL expression on cardiac macrophages remained elevated after IRI into the progression to heart failure, consistent with the AXL expression we detected on cardiac macrophages during ischemic cardiomyopathy and the increased AXL expression observed in myocardial extracts from heart failure patients (13). Elevated levels of the AXL ligand Gas6 have also been observed in heart failure patients (14).

A survey of tissue-resident macrophages in a variety of tissues revealed widespread expression of AXL on macrophages throughout the body, consistent with macrophage profiles within the ImmGen databases. In addition to its inflammatory role in the heart, we found that AXL regulated macrophage polarization during peritonitis, supporting the concept that AXL regulation of proinflammatory reprogramming is a conserved mechanism that may be translatable to macrophages in other inflammatory conditions, although this effect is likely to be context dependent. For example, during acute viral infection in the lung, AXL functions by clearing apoptotic cells to resolve inflammation and mediate tissue repair (31), which may subsequently impair the host response to a secondary infection (32). In contrast, models of sterile tissue injury in the lung (33) and liver (29) have demonstrated that AXL promotes detrimental tissue fibrotic responses. These previous studies relied on global deletion or blockade of AXL and given that $A x l$ gene expression is not restricted to macrophages and is active on other cell types, including endothelial cells, fibroblasts, and 
dendritic cells, it is unclear whether the varied outcomes reflect the cumulative inhibition of cell-specific AXL protein function. In our studies, we leveraged mice with specific deletion of $A x l$ within the myeloid compartment and found that Axl deletion in macrophages recapitulated many of our findings in mice with global $A x l$ deletion, revealing a direct role for AXL in macrophage inflammation after reperfused infarction.

AXL signaling in macrophages exacerbated myocardial inflammation through augmentation of TLR4 signaling to enhance proinflammatory IL-1 $\beta$ production. TLR4 is known to cross signal with other receptor tyrosine kinases, including the epidermal growth factor receptor (EGFR) (34), which is required for TLR4-dependent downstream signaling cascades that lead to proinflammatory cytokine production. Furthermore, there is considerable overlap between AXL signaling cascades and canonical TLR4 downstream effectors, including NF-kB, PI3K/Akt, and TBK1 (35-37), suggesting that the molecular mechanisms regulating crosstalk between AXL and TLR4 select for specific downstream effectors to determine functional outcomes. Although a proximity ligation assay suggested close protein interactions between AXL and TLR4, AXL was dispensable for NF- $\kappa$ B signaling after TLR4 stimulation in macrophages. Instead, AXL augmented STAT1 activation after TLR4 stimulation to direct a HIF- $1 \alpha$-dependent switch to glycolytic metabolism in cardiac macrophages, and secretion of proinflammatory IL-1 $\beta$. Both STAT1 and HIF- $1 \alpha$ have been linked to M1 macrophage activation within the M1/M2 macrophage polarization paradigm (38), supporting an upstream role for AXL in the activation of inflammatory macrophage polarization after TLR4 stimulation. This was in contrast to a previous report which found that AXL signaling in peritoneal macrophages inhibited IL-1 $\beta$ through autophagy induction (39). However, this inhibitory effect was independent of STAT1 signaling. This suggests our findings in BMDMs in vitro and cardiac macrophages in vivo represent a separate and distinct pathway. Interestingly, HIF-1 $\alpha$ has been shown to directly regulate $A x l$ expression in cancer cells (40), suggesting a putative mechanism in which AXL activation in cardiac macrophages during the acute phase after reperfused infarction stabilizes HIF$1 \alpha$ to further enhance AXL-dependent inflammatory macrophage responses and promote heart failure progression.

The maladaptive role of AXL contrasts with the cardioprotective role of fellow TAM member MerTK in the infarcted heart, consistent with AXL and MerTK differentially regulating cardiac macrophage function. In support of opposing roles for AXL and MerTK, previous studies have revealed protective and detrimental roles for MerTK and AXL, respectively, at the tissue level in experimental models of immune-mediated nephritis (41) and chronic liver disease (16). Here, we report at the cellular level that AXL signaling promotes proinflammatory macrophage activation, as $A x l$ deficiency reduced proinflammatory $\mathrm{MHCII}^{\text {hi }}$ cardiac macrophages after IRI. Similarly to macrophages within atherosclerotic lesions (42), this occurred independently of changes in efferocytosis, as AXL was dispensable for macrophage efferocytosis of apoptotic cardiomyocytes after IRI. Macrophage polarization is linked to metabolic reprogramming and we found that AXL was required for the switch to glycolytic metabolism to fuel inflammatory responses. In contrast, MerTK is required for antiinflammatory macrophage reprogramming, as Mertk deficiency skews cardiac macrophages from an antiinflam- matory $\mathrm{MHCII}^{\text {lo }}$ to a proinflammatory $\mathrm{MHCII}^{\text {hi }}$ phenotype (12). Although macrophages utilize efferocytic metabolites to fuel mitochondrial metabolism and antiinflammatory reprogramming (43), whether MerTK is linked to this metabolic reprogramming is the subject of future investigations. Taken together, our results show that the divergent roles for AXL and MerTK in cardiac macrophages within the infarcted heart have important implications for the study of TAM receptors in both homeostasis and disease. Many studies have employed mice deficient in both $A x l$ and Mertk, which may yield equivocal results if one receptor dominates the phenotype or the receptors diverge in function. Our results necessitate examining TAM receptor function both in isolation and combination.

In a therapeutic context, our findings suggest that clinical AXL inhibition in the acute phase after MI may reduce inflammation and adverse ventricular remodeling after MI to limit the progression to heart failure. The link between AXL and macrophage secretion of proinflammatory IL- $1 \beta$ indicates that AXL inhibition may be an interesting alternative to canakinumab, the monoclonal antibody targeting IL-1 $\beta$ that demonstrated efficacy in reducing recurrent cardiovascular events in humans but failed to gain approval for cardiovascular disease indications. The specific and highly selective AXL inhibitor used in our studies is orally bioavailable and has demonstrated favorable safety profiles and activity over prolonged periods of administration in clinical trials for the treatment of cancer in humans (44). Targeted delivery of an AXL inhibitor to cardiac macrophages during the perioperative period would circumvent the differential role AXL may exert in different myocardial cell populations. However, our data in whole-body Axl knockouts and mice treated with the AXL inhibitor suggest that transient inhibition of AXL confers protection with minimal off-target effects. Although simultaneous inhibition of AXL signaling and preservation of MerTK function led to greater cardioprotection, AXL may exert dominance over MerTK in the response to IRI, as combined loss of $A x l$ and Mertk or treatment of Mertk-deficient mice with the AXL inhibitor improved cardiac repair. Given the potential dominance of AXL over MerTK and the relative ease in therapeutic targeting of AXL compared with MerTK cleavage, strategies that inhibit AXL may yield more immediate clinical benefits in cardiovascular disease.

In conclusion, our findings reveal that AXL aggravates cardiac repair by directing proinflammatory metabolic reprogramming of macrophages and that the mechanism is distinct from MerTK, the latter of which is necessary for macrophage efferocytosis of apoptotic cardiomyocytes to initiate inflammation resolution. These findings support exploration of leveraging macrophage TAM receptors in humans to promote inflammation resolution and limit adverse ventricular remodeling that leads to heart failure.

\section{Methods}

Human ischemic cardiomyopathy specimens. Cardiac tissue specimens were obtained from the explanted hearts of adult patients with ischemic cardiomyopathy undergoing cardiac transplantation at Northwestern Memorial Hospital. Explanted hearts were immediately immersed and rinsed with cold cardioplegia solution. Tissue specimens from infarct-scar or viable remote (absence of fibrosis) myocardium were obtained from the lateral wall of the left ventricle. Specimens were maintained in cold cardioplegia solution to preserve tissue 
integrity. Within 1 hour of procurement, specimens were digested with collagenase type II (600 U/mL, Worthington) and DNase I (0.1 mg/ $\mathrm{mL}$; Sigma-Aldrich) in HBSS at $37^{\circ} \mathrm{C}$ for 30 minutes with agitation. Specimens were subsequently triturated through a $40-\mu \mathrm{m}$ cell strainer to prepare a single-cell suspension for analyses by flow cytometry.

HUman STEMI plasma specimens. Subjects were excluded from the study if they were pregnant or unable to consent. Human peripheral blood was collected in BD Vacutainer EDTA-coated tubes (lavender top) from healthy controls or patients diagnosed with STEMI via electrocardiography within 24 hours of being admitted to the cardiac care unit at Northwestern Memorial Hospital. Blood was centrifuged at $2,800 \mathrm{~g}$ for 15 minutes at $4^{\circ} \mathrm{C}$ to remove cells and platelets and plasma was stored at $-80^{\circ} \mathrm{C}$ until analyses. Levels of solAXL in diluted plasma (1:100) were measured by ELISA according to the manufacturer's instructions (R\&D Systems). Absorbances were read at $450 \mathrm{~nm}$ on an iMark Microplate Reader (BIO-RAD).

Mice. C57BL/6J mice were bred in our animal facility prior to use as WT controls. MerKD (referred to herein as $\mathrm{Merth}^{-/-}$), cleavage-resistant MerTK (Mertk $\left.{ }^{C R / C R}\right)$, and $A x^{-/-}$mice have been previously described $(12,45,46)$ and were backcrossed to C57BL/6J for 10 generations. $\mathrm{Mertk}^{-/-}$and $\mathrm{Axl}^{-/-}$mice were crossed to generate mice double-deficient

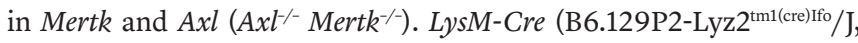
stock no. 004781), $\alpha$ MHC-mCherry (Tg(Myh6*-mCherry)2Mik, stock no. 021577), and Stat1 ${ }^{-/}$(B6.129S(Cg)-Stat ${ }^{\text {tm1Dlv} / J, ~ s t o c k ~ n o . ~ 012606) ~}$ mice were purchased from The Jackson Laboratory. $A x^{[l / f l}$ mice were generated in the C57BL/6J background as previously described (19) and provided by Carla Rothlin (Yale University, New Haven, Connecticut, USA). $A x^{[t / f l}$ mice were crossed with mice expressing $L y s M$-Cre to generate mice with specific deletion of $A x l$ in myeloid cells $\left(\mathrm{LysM}-\mathrm{Cre}^{+}\right.$ $A x l^{f / f l}$ mice). $A x l^{[l / f l}$ littermates without LysM-Cre were used as controls in experiments with $L y s M-C r e^{+} A x l^{l / f l}$ mice. Mice were housed in temperature- and humidity-controlled, pathogen-free environments and kept on a 14-hour/10-hour day/night cycle with access to standard mouse chow and water ad libitum. Two- to 4-month-old female or male mice were used for experiments.

AXL cleavage-resistant mice. Cleavage-resistant AXL protein was expressed from recombinant cDNA clones, which were initially screened in vitro. CRISPR/Cas9 targeted mutagenesis and deletion of CCCCCACCTCGCGCCTTC between exons 10 and 11 of murine $A x l$ in the C57BL/6J background was performed in collaboration with the Transgenic and Targeted Mutagenesis Laboratory at Feinberg School of Medicine at Northwestern University. This specifically deleted a 6-amino acid region within a 14-amino acid stalk region proximal to the transmembrane domain of AXL to generate cleavage-resistant AXL mice $\left(A x l^{C R / C R}\right)$. The male founder, Axl-Del2 number 6598, was born on July 18, 2017 and genotyping confirmed the presence of the correct allele. PCR primer oligonucleotides for A $x l^{C R / C R}$ genotyping were as follows: Axl-saF, ACTCACTGGTCATTCCACACC and Axl-saR, CCATGACTTCAGCTTCCCCG. See Supplemental Methods for full genotyping protocol.

Myocardial reperfused infarction. Surgeries were performed on female mice at 2-4 months of age. Mice were anesthetized with Avertin (2,2,2-tribromoethanol, 99\%, $0.1 \mathrm{mg} / \mathrm{kg}$ i.p.; Alfa Aesar) and received sustained-release buprenorphine $(0.1 \mathrm{mg} / \mathrm{kg}$ s.c.; ZooPharm) prior to the first incision. Puralube Vet Ointment (Dechra) was applied to the eyes and mice were secured in a supine position and endotracheally intubated to an Inspira Advanced Safety Single Animal Pressure/Vol- ume Controlled Ventilator (Harvard Apparatus). Animals were maintained at $37^{\circ} \mathrm{C}$ using a far-infrared warming pad (Kent Scientific) and animal temperature was monitored throughout surgery by a rectal probe using a MircoTherma 2 meter (ThermoWorks). Using a Leica S4E dissecting microscope and ACE Light Source (Schott), a left thoracotomy was performed with the aid of a Geiger Thermal Cautery Unit (Delasco) to maintain normal hemostasis. The left ventricle was visualized and the proximal left anterior descending (LAD) coronary artery was temporarily ligated with Surgipro II 7-0 monofilament polypropylene sutures (Covidien) approximately $2 \mathrm{~mm}$ distal to the site of its emergence from under the left atrium. Flexible plastic tubing (Tygon) was used during ligation to preserve the integrity of the vessel. Blanching/pale discoloration and hypokinesis of the anterior wall verified ligation. During ischemia, animal anesthesia was continuously monitored and additional Avertin was administered following a positive toe-pinch response. Following 45 minutes of ischemia, the ligature was reopened to allow for reperfusion. The reopened ligature was left in place to aid future analyses of infarct tissue. Reperfusion was confirmed by restoration of blood flow in the LAD and return of color to the left ventricle. Using Surgipro II 6-0 monofilament polypropylene sutures (Covidien), the surgical site was closed in layers starting with the chest wall followed by the pectoral muscle and finally skin and subcutaneous tissue. Animals were allowed to recover on a heating pad (Sunbeam) prior to being returned to cages. Mice dying within 48 hours of surgery were treated as technical errors and excluded from analyses.

Statistics. Statistical analyses were performed with Prism 9 software (GraphPad Software). Comparisons between 2 groups were performed using 2-tailed, unpaired $t$ tests with 95\% confidence interval. For comparisons of more than 2 variables, 1-way or 2-way ANOVA was utilized with $95 \%$ confidence interval and when necessary, Tukey's test was used to correct for multiple comparisons. For in vivo experiments, experimental sample size is depicted in figures and represent pooled data from 2 or more independent experiments. For in vitro experiments, experimental sample size is depicted in figures and represent 2 or more independent experiments. Data are presented as mean \pm SEM. Criteria for significant differences $\left({ }^{*} P<\right.$ $\left.0.05,{ }^{* *} P<0.01,{ }^{* *} P<0.001\right)$ are located in figure legends. A $P$ value of less than 0.05 was considered significant. Differences labeled NS are not statistically significant.

Study approval. These studies were approved by the Institutional Review Board (nos. STU00012288 and STU00075325) at Northwestern University and performed in accordance with the Helsinki Doctrine on Human Experimentation. Written consent was obtained from all study participants. Animal studies were conducted in accordance with guidelines using a protocol approved by the Institutional Animal Care and Use Committee at Northwestern University.

A complete description of methods is provided in Supplemental Methods.

\section{Author contributions}

MD and EBT designed the research studies. MD and KG conducted experiments and acquired data. CVR generated and provided the $A x^{f / / f l}$ mice. MS and IT performed initial screenings to identify AXL cleavage-resistant mutants. LDW provided the human myocardial specimens. All authors contributed to data analyses. MD and EBT wrote the manuscript, and all authors contributed to manuscript revision. 


\section{Acknowledgments}

This work was supported by American Heart Association grant 18CDA34110032 (to MD) and NIH grants F32HL127958 (to MD), R01HL122309 (to EBT), R01HL139812 (to EBT), R35HL145228 (to IT), and R01HL127464 (to IT). The authors thank Lynn Doglio, Rajeshwar Awatramani, and Pei-Ken Hsu of the Transgenic and Targeted Mutagenesis Laboratory at Feinberg School of Medicine at Northwestern University for CRISPR/Cas9 gene editing services. We are thankful to Anna Hus- kin, Program Development Manager in the Clinical Trials Unit of the Bluhm Cardiovascular Institute and Patrick McCarthy, the Executive Director of the Bluhm Cardiovascular Institute. Publication of this research was supported by the Sidney and Bess Eisenberg Memorial Fund.

Address correspondence to: Edward B. Thorp, 300 East Superior St., Tarry 3-705, Chicago, Illinois 60611, USA. Phone: 312.503.4309; Email: ebthorp@northwestern.edu.
1. DeBerge M, et al. Macrophages in heart failure with reduced versus preserved ejection fraction. Trends Mol Med. 2019;25(4):328-340.

2. Adamo L, et al. Reappraising the role of inflammation in heart failure. Nat Rev Cardiol. 2020;17(5):269-285

3. King KR, et al. IRF3 and type I interferons fuel a fatal response to myocardial infarction. Nat Med. 2017;23(12):1481-1487.

4. Huang S, Frangogiannis NG. Anti-inflammatory therapies in myocardial infarction: failures, hopes and challenges. Br JPharmacol. 2018;175(9):1377-1400.

5. Ridker PM, et al. Antiinflammatory therapy with canakinumab for atherosclerotic disease. $N$ Engl J Med. 2017;377(12):1119-1131.

6. Zagórska A, et al. Diversification of TAM receptor tyrosine kinase function. Nat Immunol. 2014;15(10):920-928.

7. Rothlin CV, et al. TAM receptors are pleiotropic inhibitors of the innate immune response. Cell. 2007;131(6):1124-1136.

8. Cai B, et al. MerTK cleavage limits proresolving mediator biosynthesis and exacerbates tissue inflammation. Proc Natl Acad Sci U S A. 2016;113(23):6526-6531.

9. Cai B, et al. MerTK receptor cleavage promotes plaque necrosis and defective resolution in atherosclerosis. J Clin Invest. 2017;127(2):564-568.

10. Wan E, et al. Enhanced efferocytosis of apoptotic cardiomyocytes through myeloid-epithelial-reproductive tyrosine kinase links acute inflammation resolution to cardiac repair after infarction. Circ Res. 2013;113(8):1004-1012.

11. Howangyin K-Y, et al. Myeloid-epithelial-reproductive receptor tyrosine kinase and milk fat globule epidermal growth factor 8 coordinately improve remodeling after myocardial infarction via local delivery of vascular endothelial growth factor. Circulation. 2016;133(9):826-839.

12. DeBerge $M$, et al. MerTK cleavage on resident cardiac macrophages compromises repair after myocardial ischemia reperfusion injury. Circ Res. 2017;121(8):930-940.

13. Batlle $\mathrm{M}$, et al. AXL receptor tyrosine kinase is increased in patients with heart failure. Int J Cardiol. 2014;173(3):402-409.

14. Caldentey $\mathrm{G}$, et al. Serum levels of growth arrest-specific 6 protein and soluble AXL in patients with ST-segment elevation myocardial infarction. Eur Heart J Acute Cardiovasc Care. 2017;8(8):708-716.

15. Heng TSP, et al. The Immunological Genome Project: networks of gene expression in immune cells. Nat Immunol. 2008;9(10):1091-1094.

16. Zagórska A, et al. Differential regulation of hepatic physiology and injury by the TAM receptors Axl and Mer. Life Sci Alliance. 2020;3(8):e202000694.

17. Lantz C, et al. Single-cell RNA sequencing uncovers heterogenous transcriptional signatures in macrophages during efferocytosis. Sci Rep. 2020;10(1):14333

18. Batchu SN, et al. Axl modulates immune activation of smooth muscle cells in vein graft remodeling. Am J Physiol Heart Circ Physiol. 2015;309(6):H1048-H1058.

19. Schmid ET, et al. AXL receptor tyrosine kinase is required for $\mathrm{T}$ cell priming and antiviral immunity. Elife. 2016;5:e12414.

20. Chakarov S, et al. Two distinct interstitial macrophage populations coexist across tissues in specific subtissular niches. Science. 2019;363(6432):eaau0964.

21. Epelman S, et al. Embryonic and adult-derived resident cardiac macrophages are maintained through distinct mechanisms at steady state and during inflammation. Immunity. 2014;40(1):91-104.

22. Zhang W, et al. Necrotic myocardial cells release damage-associated molecular patterns that provoke fibroblast activation in vitro and trigger myocardial inflammation and fibrosis in vivo. J Am Heart Assoc. 2015;4(6):e001993.

23. Tannahill GM, et al. Succinate is an inflammatory signal that induces IL-1 $\beta$ through HIF- $1 \alpha$. Nature. 2013;496(7444):238-242.

24. Cramer T, et al. HIF-1 $\alpha$ is essential for myeloid cell-mediated inflammation. Cell. 2003;112(5):645-657.

25. Parra-Izquierdo I, et al. Lipopolysaccharide and interferon- $\gamma$ team up to activate HIF- $1 \alpha$ via STAT1 in normoxia and exhibit sex differences in human aortic valve interstitial cells. Biochim Biophys Acta Mol Basis Dis. 2019;1865(9):2168-2179.

26. Joshi VD, et al. A role for Stat1 in the regulation of lipopolysaccharide-induced interleukin-1beta expression. J Interferon Cytokine Res. 2006;26(10):739-747.

27. Holland SJ, et al. R428, a selective small molecule inhibitor of Axl kinase, blocks tumor spread and prolongs survival in models of metastatic breast cancer. Cancer Res. 2010;70(4):1544-1554.

28. Seitz HM, et al. Macrophages and dendritic cells use different Axl/Mertk/Tyro3 receptors in clearance of apoptotic cells. JImmunol. 2007;178(9):5635-5642.

29. Bárcena C, et al. Gas6/Axl pathway is activated in chronic liver disease and its targeting reduces fibrosis via hepatic stellate cell inactivation. J Hepatol. 2015;63(3):670-678.

30. Shibata T, et al. Axl receptor blockade ameliorates pulmonary pathology resulting from primary viral infection and viral exacerbation of asthma. JImmunol. 2014;192(8):3569-3581.

31. Fujimori T, et al. The Axl receptor tyrosine kinase is a discriminator of macrophage func- tion in the inflamed lung. Mucosal Immunol. 2015;8(5):1021-1030.

32. Shibata T, et al. Respiratory syncytial virus infection exacerbates pneumococcal pneumonia via Gas6/Axl-mediated macrophage polarization. J Clin Invest. 2020;130(6):3021-3037.

33. Espindola MS, et al. Targeting of TAM receptors ameliorates fibrotic mechanisms in idiopathic pulmonary fibrosis. Am J Respir Crit Care Med. 2018;197(11):1443-1456.

34. De S, et al. Erlotinib protects against LPS-induced endotoxicity because TLR4 needs EGFR to signal. Proc Natl Acad Sci US A. 2015;112(31):9680-9685.

35. Paccez JD, et al. The receptor tyrosine kinase Axl is an essential regulator of prostate cancer proliferation and tumor growth and represents a new therapeutic target. Oncogene. 2013;32(6):689-698.

36. Son B-K, et al. Gas6/Axl-PI3K/Akt pathway plays a central role in the effect of statins on inorganic phosphate-induced calcification of vascular smooth muscle cells. Eur JPharmacol. 2007;556(1):1-8.

37. Cruz VH, et al. Axl-mediated activation of TBK1 drives epithelial plasticity in pancreatic cancer. JCI Insight. 2019;4:e126117.

38. Tugal D, et al. Transcriptional control of macrophage polarization. Arterioscler Thromb Vasc Biol. 2013;33(6):1135-1144.

39. Han J, et al. Autophagy induced by AXL receptor tyrosine kinase alleviates acute liver injury via inhibition of NLRP3 inflammasome activation in mice. Autophagy. 2016;12(12):2326-2343.

40. Rankin EB, et al. Direct regulation of GAS6/ AXL signaling by HIF promotes renal metastasis through SRC and MET. Proc Natl Acad Sci U S A. 2014;111(37):13373-13378.

41. Zhen Y, et al. Opposing roles of tyrosine kinase receptors Mer and Axl determine clinical outcomes in experimental immune-mediated nephritis. JImmunol. 2016;197(6):2187-2194.

42. Subramanian M, et al. Deficiency of AXL in bone marrow-derived cells does not affect advanced atherosclerotic lesion progression. Sci Rep. 2016;6(1):39111.

43. Zhang S, et al. Efferocytosis fuels requirements of fatty acid oxidation and the electron transport chain to polarize macrophages for tissue repair. Cell Metab. 2019;29(2):443-456.

44. Loges S, et al. A first-in-patient phase I study of BGB324, a selective Axl kinase inhibitor in patients with refractory/relapsed AML and highrisk MDS. J Clin Oncol. 2016;34(15_suppl):2561.

45. Scott RS, et al. Phagocytosis and clearance of apoptotic cells is mediated by MER. Nature. 2001;411(6834):207-211.

46. Lu Q, et al. Tyro-3 family receptors are essential regulators of mammalian spermatogenesis. Nature. 1999;398(6729):723-728. 